\title{
Genetic and non-genetic clonal diversity in cancer evolution
}

2

3

4

5

6

7 9

10

11

12

†email: nicholas.mcgranahan.10@ucl.ac.uk

10

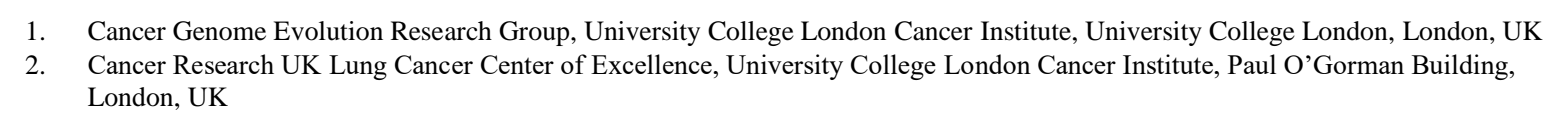




\section{$44 \quad \underline{\text { Abstract }}$}

45

46

47

48

49
The observation and analysis of intra- tumour heterogeneity (ITH), particularly in genomic studies, has advanced our understanding of the evolutionary forces that shape cancer growth and development. However, only a subset of the variation observed in a single tumour will have an impact on cancer evolution, highlighting the need to distinguish between functional and non- functional ITH. Emerging studies highlight a role for the cancer epigenome, transcriptome and immune microenvironment in functional ITH. Here, we consider the importance of both genetic and non- genetic ITH and their role in tumour evolution and present the rationale for a broad research focus beyond the cancer genome. Systems- biology analytical approaches will be necessary to outline the scale and importance of functional ITH. By allowing a deeper understanding of tumour evolution this will, in time, encourage development of novel therapies and improve outcomes for patients. 


\section{Introduction}

In 1859, in his seminal text On the Origin of Species, Charles Darwin described a theory of branching evolution by natural selection, based on observations of incredible diversity of phenotypes amongst animals ${ }^{1}$. At the time, the mechanisms underpinning such diversity, including genetic recombination and mutation, were unknown, but such were the strength of his deductions about the function of these variations that this theory remains in the scientific mainstream today.

Cancer is also an evolutionary process ${ }^{2,3}$, and it was the observation of phenotypic heterogeneity within tumours that led Nowell to hypothesise that Darwinian clonal evolution underpinned their development ${ }^{2}$. Since then, 'intra-tumour' heterogeneity (ITH), describing diversity within individual tumours, has been defined at multiple different levels, including single point mutations, somatic copy number alterations (SCNAs), epigenetic and transcriptomic changes influencing gene expression, the antitumour immune response and other features of the tumour microenvironment.

An important task that remains is to distinguish between 'functional' variation, conferring a fitness effect that brings about an important change in tumour phenotype, from 'non-functional' variation ${ }^{4}$ (Figure 1). Indeed, the extent to which ITH is a result of the stochastic accumulation of mutations following the acquisition of founding 'driver' events, rather than the result of continual clonal evolution and selection through time and space remains an open scientific question and a topic of debate ${ }^{5}$. It is likely that the true extent of selection during cancer evolution varies both between cancer types, and within individual cancers of the same type (Box 1).

This Review will explore what is known about functional and non-functional ITH in cancer, outlining unresolved areas of debate that warrant further study. It will address the diverse causes of ITH and how this might impact cancer treatment and prognosis. The advent of high throughput multi-omics has increased our understanding of the interplay between different cellular processes that contribute to $\mathrm{ITH}^{6}$. In order to appreciate in full the importance of ITH in cancer, we must interrogate more than just mutations, SCNAs, or gene expression in isolation; rather, we must seek to link all factors that may influence tumour phenotype. With a systems-biology lens such as this, we may gain the resolution required to better understand cancer evolution and comprehend its origins and vulnerabilities.

\section{$\underline{\text { ITH and evolution }}$}

Cancer develops through clonal evolution ${ }^{2,3}$. Genetic variation acts as a primary substrate for this evolution. This arises through different mechanisms of genomic instability, including endogenous and exogenous processes that generate point mutations, as well as chromosomal instability $(\mathrm{CIN})^{7,8}$.

Early studies of tumour heterogeneity developed the notion of cancer as an evolutionary, as well as a genetic, disease. Interphase fluorescence in-situ hybridisation and karyotyping of metaphase chromosomes in the 1990s demonstrated the presence of multiple clones within a tumour ${ }^{9,10}$. Comparative genomic hybridisation microarray analysis enabled accurate characterisation of the copy-number profiles of cancer clones and expanded on these findings. For example, Navin and colleagues classified breast tumours as either 'monogenomic', containing a population of near-homogeneous tumour cells with analogous genomic profiles, or 'polygenomic', containing subpopulations or 'clones' with distinct genomic profiles, and demonstrated that clones in polygenomic tumours were descended from a common ancestor by branched evolution ${ }^{11}$. The advent of next-generation sequencing has enabled this to be characterised with finer granularity. In a seminal study in 2012, 
141 Gerlinger and colleagues performed multi-region whole-exome sequencing on tumour putative driver mutations within the same tumour, and ongoing branched evolution over time $^{12}$.

Since these early observations, a central focus of research has been to distil functional from non-functional somatic variation and to characterise the strength of the evolutionary forces that shape tumour development over time. However, the extent to which cancer is under continuous selection during its development is a contentious topic in the field, echoing a long-standing debate in evolutionary biology ${ }^{13}$. Observed differences between parts of the same tumour do not themselves indicate the presence of competing subclones which are under selection. The accumulation of random somatic alterations over time means that genotypes will diverge even in the absence of selection pressures. As such, some studies have suggested that a subset of tumours may evolve neutrally following the acquisition of necessary driver events ${ }^{14-18}$.

Low ITH of driver events, potentially indicative of selective 'clonal sweeps' of certain phenotypes early in tumour evolution, is well-described in multiple cancer types ${ }^{19-23}$. Conversely, other studies have highlighted the presence of subclonal driver mutations in cancer genes, whereby only a subset of cancer cells, or clones, harbour functional somatic events assumed to confer a fitness advantage ${ }^{24-29}$.

\section{Methods for evaluating ITH}

Accurately recapitulating the evolutionary history of a tumour from a genomic snapshot, typically provided at a single time point, and frequently from a single part of a tumour, can be problematic. Despite consensus that one size may not fit all where tumour evolution is concerned, non-uniform terminology and methods of analysis continue to hamper efforts to classify tumours by their patterns of evolutionary development.

Many studies, such as a seminal analysis of 21 breast cancer genomes by Campbell and colleagues ${ }^{30}$, perform bulk sequencing on a single sample and attempt to infer the evolutionary history of the tumour from the variant allelic frequencies (VAF) of somatic mutations. Such studies must attempt to account for variables such as the amount of nontumour tissue sampled, the SCNA profile of the tumour, as well as the accuracy and depth of sequencing, all of which can confound accurate interpretation of the data. Another problem among such studies is sampling bias; a variant may be present in all cancer cells sampled, but not all cancer cells in the tumour (Figure 2). In addition, it may not be possible to distinguish the VAF of mutations within subclones that are the product of selection from those within the VAF distribution 'tail' which is a feature of neutral evolution ${ }^{16,31,32}$. This tail-like distribution of read counts of passenger mutations that are not under selection reflects random mutagenesis at each cell division and the expected relationship between the number of mutational events and their clonal frequency over time.

Multi-region sampling, while it does not mitigate against neutral tails, may help to identify and classify subclones more accurately and reduce sampling bias. Nevertheless, this does not represent a 'silver bullet'; typically, only a tiny fraction of the total tumour is assessed $^{33}$. Sequencing of every single tumour cell, which remains impossible, would be required to resolve this definitively. Another option is representative sequencing, whereby homogenised fixed tumour material that was not used for pathology is sequenced, thus reducing sampling bias and misclassification rates $^{33}$.

Even if clonal sweeps are accurately defined, distinguishing between such an event that may have occurred in the recent past, and one that was present at the inception of the tumour is not always possible. Furthermore, negative selection that has eliminated a clone 
prior to sampling is not detectable using VAF-based approaches. Therefore, other methods are required to disentangle the temporal order of events and to distinguish functional events, subject to selection, from non-functional events, which are not.

Copy-number gains and whole-genome duplication events may be used to time somatic alterations and separate early from late events occurring during tumour evolution ${ }^{29,34}$. In the Pan Cancer Analysis of Whole Genomes (PCAWG), this approach enabled inference of trends in clonal architecture across tumour types ${ }^{29}$. In colorectal adenocarcinoma, for instance, mutations in APC, KRAS and TP53 were shown to be predominantly early events, while certain copy-number alterations including 15q, 21q and 22q loss, were generally late ${ }^{29}$.

A method borrowed from evolutionary biology can provide orthogonal evidence of clonal selection. $\mathrm{dN} / \mathrm{dS}$, an assessment of the ratio of substitution rates at nonsynonymous vs. synonymous sites, revealed substantial selection pressures acting on apparently normal tissue $^{35}$. In cancer, $\mathrm{dN} / \mathrm{dS}$ has also been used to reveal positive selection globally, and within specific cancer genes, as well as a near-absence of negative, or purifying selection ${ }^{36}$ (Figure 3). Whilst dN/dS provides information on global patterns of selection, particularly when large numbers of samples are analysed, it is nevertheless unable to infer selection within specific elements of interest in individual clones (Figure 3C). In addition, further research is needed to enable accurate measurement of selection in the context of other events such as non-coding mutations, indels or structural variants.

Robustly describing tumour evolution with a single binary label, such as neutral or branched, punctuated or gradual ${ }^{37}$, remains problematic. Indeed, separate analyses of identical data can lead to disparate conclusions: a comparison of VAFs in 904 cancers from 14 cancer types, including a minority subjected to multi-region sampling, by Williams and colleagues suggested that the subclonal VAF distributions in 36\% (323/904) of these could be explained by neutral evolution ${ }^{16}$, but subsequent work using an orthogonal approach (dN/dS), found evidence of subclonal selection within cancer genes in these 323 tumours ${ }^{16,38}$.

Single-cell sequencing platforms may provide further insight into tumour evolution (reviewed elsewhere ${ }^{39}$ ). This approach presents the opportunity to analyse cellular epigenomes, as assessed through DNA methylation, histone configuration or chromatin accessibility, and cell states, inferred from transcriptomic or protein expression data. This enables explicit evolutionary context to be added to epigenetic or transcriptomic events. Lineage reconstruction at the single cell level has the potential to improve vastly our understanding of tumour phylogeny. An exemplar of this is Direct Library Preparation singlecell whole-genome sequencing, which allows for identification of clonal populations of single cells, pinpointing unique aspects of their genomes ${ }^{40}$. This enables aggregation into 'pseudo-bulk' samples from which clonal phylogeny may inferred. At present, however, the accuracy of methods to call mutations from typically shallow single-cell sequencing may be limited by PCR errors and allelic dropout. Efforts to resolve this issue will enhance the future success of this approach.

\section{[H1]Copy-number ITH}

So far, the main form of variation discussed in this review has been point mutations. However, CIN, and structural variation including SCNAs, whole genome duplication and chromothripsis events may engender large fitness effects. The extent to which CIN provides the substrate for ongoing selection and branched evolution in cancer is unclear. Although CIN has long been linked to poor prognosis ${ }^{7}$, research has only recently explored the question of whether this process continues throughout tumour evolution, or, conversely, whether SCNAs are predominantly relics of past genomic instability. A single-cell study of 12 triple- 
negative breast cancers identified aneuploidy as a punctuated, early event in tumour evolution, preceding clonal expansion of a population of cells with a stable karyotype ${ }^{20}$. This finding was supported by recent work on colorectal cancer reported in a preprint; Cross et al studied CIN within 755 samples, taken from multiple tumour regions or longitudinally in 167 patients and found that certain dominant SCNAs were established early in tumour evolution and persisted through negative selection of karyotype diversity, in spite of ongoing CIN through therapy administration and progression to metastasis ${ }^{41}$.

There are also examples of CIN generating functional events late in tumour evolution. For example, PCAWG demonstrated that chromosomal gains occur throughout 'molecular time', from early human development to the final stages of tumour growth (median molecular time 0.60; IQR 0.10-0.87). However, within certain cancer types such as lung cancer, papillary renal cancer and melanoma, they are predominantly late events ${ }^{29}$. Furthermore, in a selection and was associated with metastatic progression, as evidenced by the fact that it was a clonal event in just $26 \%$ of primary tumour samples versus $64 \%$ of metastases ${ }^{42}$. Late armlevel SCNAs have also been described in other cancer types ${ }^{43-45}$.

Watkins et al. interrogated pan-cancer multi-region data to assess the degree to which CIN provides a substrate for subclonal phenotypic diversification ${ }^{46}$. This work found evidence of parallel evolutionary events, in which the same genes were affected by different subclonal SCNAs in 37\% of tumours analysed; examples included gains at 1q21.3-q44 which encompasses BCL9, MCL1 and ARNT, at 5p15.33 which contains TERT, and at 8q24.1 which contains $M Y C$. Independent analysis of metastases by multi-region sampling revealed subclonal events within certain tumour types, including gains to MYC in ccRCC and CCNDI in $H E R 2^{+}$breast cancer, which were enriched in metastases relative to primary tumours. Together, this suggests that late in tumour evolution, CIN engenders extensive subclonal diversity. It also expands on in vitro work within patient-derived tumour organoids, where ongoing CIN led to karyotypic heterogeneity over time in models of colorectal carcinoma ${ }^{47}$.

It is likely that, even when looking at the mutation and copy number landscapes in parallel, we still fail to capture events with a large influence on cellular fitness and may draw imperfect conclusions as a result. Extending our focus beyond the genome to the epigenome, transcriptome, and the immune microenvironment may allow us a greater understanding of the true extent of ITH. In this way, we can more accurately define the functional evolutionary processes that influence phenotype (Figure 5).

\section{[H1]Epigenetic and transcriptomic ITH}

Epigenetic dysregulation influences gene expression and is widespread in cancer, occurring by varied mechanisms such as promoter hypermethylation, altered enhancer activity, and changes in chromatin configuration ${ }^{48}$.

Alterations to the cancer epigenome can be binary, functioning as 'on or off' switches, or induce transient changes in gene expression, forming part of highly plastic gene expression networks. They but can also govern copy number changes. For example, the interplay between lysine demethylases and methyltransferases and their relative activity upon histone $\mathrm{H} 3$ lysine 4 (H3K4), H3K9 and H3K27 has been shown to affect copy-number amplification of the EGFR oncogene, which encodes the epidermal growth factor receptor ${ }^{49}$. Dysregulation of the cancer epigenome may be global in some cancers. In a pan-cancer analysis, increased global enhancer expression was seen across multiple cancer types, and enhancer activity correlated with the fraction of the genome affected by SCNAs ${ }^{50}$. This intriguing potential consequence of CIN was posited to relate to its impact on chromatin 
state, although the evolutionary context of such epigenomic dysfunction within tumours requires further elucidation. Interestingly, mutations in genes encoding epigenetic modifiers alone are sufficient to drive some, and an analysis of 24 types of childhood cancer found this group of genes, including KMT2C, KMT2D and SMARCA4, to be the most commonly mutated (mutations in epigenetic modifiers affected $25 \%$ of tumours) across all molecular subtypes $^{51}$.

Like the epigenome, the cellular transcriptome is frequently aberrant in cancer. Various mechanisms, such as alternate splicing, alternate promoter usage, gene fusions and aberrant oncogenic signalling can underpin this phenomenon ${ }^{52-56}$. The impact of genetic alterations on transcription was explored extensively using bulk whole-genome sequencing with matched RNA-sequencing in the recent PCAWG analysis ${ }^{55}$. In this analysis, SCNAs emerged as the dominant genomic event influencing gene expression, contributing to $17 \%$ of gene expression variation, compared to somatic and germline genetic variation in cis, which contributed to $1.8 \%$ and $1.3 \%$ respectively. Cumulatively, non-coding mutations contributed more to variation in allelic expression than coding mutations ${ }^{55}$. This underscores the potential problems of restricting focus to exonic mutations, and is supported by our own analysis of lists of cancer genes (Figure 4). We compared cancer genes identified by Bailey et al ${ }^{57}$, who systematically catalogued mutations from 9,423 tumour exomes, with those identified in the COSMIC Cancer Gene Census ${ }^{58}$, which incorporates evidence of functional involvement alongside increased mutation frequency when curating cancer gene lists. As would be expected, a whole-exome sequencing approach only identifies a subset (330/657) of COSMIC cancer genes. Intriguingly, it fails to identify the majority of genes affected by translocations and amplifications (Figure 4A). Greater proportions of the COSMIC cancer genes affecting certain cancer types (BRCA, LGG) are identified by Bailey than others (PAAD, SARC) (Figure 4B). This highlights that many cancer genes are likely undiscovered, a substantial proportion of which may be driven by mechansisms beyond point mutations. For example, RNA variants, generated by editing enzymes, are an additional source of diversity within tumours that impact upon protein function in cancer and would be missed by whole-exome sequencing 59,60

The importance of transcriptomic variation in cancer is underlined by the ability of expression-based biomarkers to predict clinical outcome ${ }^{61}$. However, the evolutionary context of this variation is also important: considering ITH in gene expression through multiregion bulk RNA-sequencing can significantly improve the predictive ability of such biomarkers. For example, in non-small cell lung cancer (NSCLC), a prognostic gene expression signature calculated from clonally expressed genes reduced the impact of sampling bias, a problem also highlighted by a transcriptomic analysis of multifocal prostate cancer $^{62,63}$. Multi-region transcriptomics can also shed light on other tumour evolutionary processes. Biswas et al underlined the dominant role of SCNAs in influencing gene expression, as ITH of SCNAs was strongly correlated to ITH of gene expression ${ }^{62}$. A multiregion transcriptomic analysis of four patients with advanced bladder cancer identified distinct molecular subtypes thought to arise from different urothelial progenitors in distinct regions of the same tumour, suggesting that tumour subtypes may be somewhat plastic rather than entirely pre-determined ${ }^{64}$.

An important caveat for bulk transcriptomic and epigenetic analysis is that such datasets comprise tumour and stromal gene expression which, currently, we can only partly deconvolve; tumour purity may therefore introduce bias into transcriptomic analysis of $\mathrm{ITH}^{65}$. Integrated analysis of the genome and transcriptome may help to deconvolve tumourspecific gene expression within bulk sequencing samples ${ }^{66}$.

Single-cell sequencing can capture epigenomic and transcriptomic changes and has helped to shape the concept of cellular transcriptomic promiscuity and its influence on 
phenotypic plasticity ${ }^{67}$. In lung adenocarcinoma, a highly plastic cellular state is associated with poor prognosis in humans and with treatment resistance in mice; this plastic state mediates transition to more diverse phenotypes and may explain ITH in some tumours ${ }^{68}$. Jacks and colleagues studied the epigenome of single lung adenocarcinoma cells en route to metastasis and revealed important changes in chromatin state which characterise gradual loss of cellular identity, and may be controlled by key transcription factors such as those of the RUNX family ${ }^{69}$. The identification of a gene expression signature of this subpopulation that associated with survival was particularly intriguing ${ }^{69}$; the ability to robustly obtain from bulk sequencing evidence of cancer cells with features of stemness, diverse transcriptional landscapes and the ability to influence the evolutionary trajectory of a tumour in the presence of selective pressures, would be of great value in both the research and clinical settings.

In studies of haematopoiesis, differences in stemness, cellular states, gene expression profiles and enhancer activity have been explicitly linked to mutations in DNA methylation genes, underscoring the importance of these genes in cancer ${ }^{70}$. However, in general, the heterogeneity of epigenetic events in human cancers, as well as their interplay with the cancer genome and transcriptome, remains poorly understood. This problem is illustrated by debates surrounding evolutionary trajectories in pancreatic cancer, where genetic driver mutations in TP53, KRAS, CDKN2A or SMAD4, when present, are almost always clonal ${ }^{71}$. In isolation, this might indicate an absence of subclonal selection in this disease, but study of the epigenome in pancreatic cancer evolution suggests that widespread chromatin remodelling might provide the substrate for selection in metastasis ${ }^{72}$. In chronic lymphocytic leukaemia, the epigenetic landscape is significantly disrupted, driving variety in cellular phenotype, and different cancer cell populations may have highly disparate epigenomes ${ }^{73}$.

Hua et al. explicitly compared the ITH of point mutations, SCNAs and DNA methylation in a multi-region study of 84 lung adenocarcinomas ${ }^{74}$. They found that tumour evolutionary trees inferred from SCNAs and DNA methylation were highly similar, demonstrating that patterns of cancer evolution may be agnostic of variant mechanism. Congruency of genomic and epigenomic evolution was also recently described in papillary renal cell carcinoma ${ }^{75}$. Future work should seek to devise tools to define more clearly the relationship between mutations, CIN and epigenetic and transcriptomic states, in both space and time. Integrating whole-genome sequencing with multi-omics is likely to be important to this endeavour.

Ultimately, mutations, SCNAs, epigenetic alterations and transcriptional alterations all influence the abundance, structure and function of proteins, the true arbiters of cellular phenotype. Therefore, proteomic studies may be critical to integrating this information. Moreover, a proteogenomics approach facilitates deep analysis of the impact of functional mutations at the pathway level. Such studies have described novel consequences of mutations and SCNAs in breast cancer ${ }^{76}$, including the identification of alterations in enzymatic activity which were not visible at the transcriptomic level, as well as in gastric and ovarian cancer ${ }^{77,78}$. In addition, recent publications studying the lung adenocarcinoma proteome with mass spectrometry and phosphoproteomics are an important development in this space ${ }^{79-81}$. Proteogenomic analyses have also helped show that the relationship between SCNAs and mRNA and protein abundance may be inconsistent. Intruiguingly, in breast cancer, genes in which a correlation was observed between SCNAs and mRNA as well as between SCNAs and protein levels were more likely to be cancer genes than those without a correlation between SCNAs and protein levels ${ }^{76}$. This suggests that in some settings multi-omic approaches that consider the proteome may be more powerful to detect functional events that those that do not. It is hoped that future studies of proteomic ITH, as well as other systemsbased approaches, will provide insight into functional clonal diversity, and help to resolve long standing debates about cancer biology. 


\section{[H1]Microenvironmental ITH}

A convincing argument to move forward from the reductionist, exclusively genomic, view of tumour evolution stems from the success of immunotherapies that disrupt signalling between cancer and immune cells. If our aim is to understand cancer evolution, we cannot ignore the environment in which a cancer cell evolves. Whether variation is functional or non-functional can be highly context dependent, depending on both the genomic background into which a mutation arises, but also the environment itself. This environmental context has an immune, and a non-immune arm.

\section{[H2] Evolutionary constraints imposed by the anti-tumour immune response}

A critical component of the tumour microenvironment in a growing cancer is the immune infiltrate. Human cellular biology has evolved to combat the threat of cancer in its tissues through a high-fidelity anti-cancer immune response ${ }^{82}$. A prominent part of this response is the adaptive immune response. Here, a tumour, or its associated non-host peptides (neoantigens) are identified and eliminated through the clonal expansion of a highly specific T-cell population and degradation by cytotoxic CD8+ T-cells, or by a CD4+ T-celldependent cytotoxic response e $^{83,8485}$.

The anti-tumour immune response equates to a clone-specific negative selection pressure and there are a number of mechanisms that cancers can co-opt in order to evade detection and elimination. Many of these are genomic. For example, mutations in beta-2microglobulin, $B 2 M$, a component of the Major Histocompatibility Complex, disrupt antigen presentation in response to immune predation ${ }^{86}$. Similarly, cancer cells undergo loss-ofheterozygosity (LOH) of the Human Leucocyte Antigen (HLA) locus on chromosome 6p. In one study, HLA LOH was detected in $40 \%$ of NSCLC and was subclonal in $65 \%$ of cases ${ }^{87}$. This finding was supported by a recent pan-cancer analysis of multi-region sampled tumours, where $22 \%$ of all tumours demonstrated subclonal loss of two copies of the same HLA allele after whole genome doubling ${ }^{46}$. SCNAs outside of the HLA locus can also promote immune evasion, for example through copy-number loss of neoantigens capable of stimulating a functional $\mathrm{T}$ cell response ${ }^{88,89}$. In mouse models of ovarian cancer, immune-excluded tumour regions were characterised by copy-number amplification of MYC target genes and increased WNT signalling ${ }^{90}$. An abundance of non-specific SCNAs has also been proposed as a predictor of poor response to immunotherapy ${ }^{91}$; however, disentangling their well-described prognostic role from a predictive one is difficult, and requires further study. There is also evidence of transcriptional neoantigen depletion underpinning immune escape. In NSCLC, downregulation of neoantigenic transcripts was found to occur via promoter hypermethylation (seen in $23 \%$ of silenced neoantigen-containing genes versus $11 \%$ of the same non neoantigen-containing genes) and was enriched in immune-infiltrated tumours with an intact HLA allele, suggestive of diverse cellular responses to the negative selection pressure imposed by the anti-tumour immune response ${ }^{89}$. Indeed, the remaining repressed neoantigens not subject to promoter hypermethylation may be affected by mechanisms that are yet to be elucidated.

Not all neoantigens stimulate a uniform anti-tumour immune response, demonstrating the importance of distinguishing between functional and non-functional ITH. Clonal neoantigens stimulate anti-tumour immunity, and those tumours containing neoantigenreactive tumour-infiltrating lymphocytes (TILs) are associated with better outcome ${ }^{92}$. In a multi-region study of NSCLC, Chain and colleagues reported a correlation between the number of T-cell clones found in all tumour regions, and the number of clonal, but not 
subclonal mutations, emphasising the importance of neoantigens in stimulating the immune response early in tumour evolution ${ }^{93}$. Furthermore, the clonality of T-cells within a tumour has also been associated with improved response to anti-programmed cell death 1 (PD1) therapy in melanoma ${ }^{94,95}$, although other facets of the T-cell repertoire, such as the diversity of circulating T-cell clones in peripheral blood, also impact response to immune checkpoint blockade ${ }^{96}$. Importantly, the clonal diversity of neoantigens can also influence the antitumour immune response. Using an immune-competent mouse model of melanoma, Wolf and colleagues showed a correlation between increased clonal diversity and ineffective rejection of the developing tumour ${ }^{97}$. This builds on earlier findings in mice which suggested that the fraction of cells expressing a clonal, immunogenic peptide is key in determining whether a tumour is eliminated, with small subclones being more able to evade immune rejection ${ }^{98}$.

As clonal diversity can influence the immune response to a developing tumour, so this response can provide a negative selection pressure on a growing tumour and in turn shape its clonal composition. This is illustrated by recent work in a glioma model, where the immune editing that occurred within immune-competent mice led to the formation of tumours with lower clonality ${ }^{99}$. In high-grade serous ovarian cancer, tumour regions with the highest levels of immune infiltrate were characterised by neoantigen depletion, subclonal HLA LOH, and low clone diversity, indicating predation of that region prior to sampling ${ }^{100}$. These findings are consistent with a study of metastatic colorectal cancer, where those metastases that persist were the least immunogenic, and harboured diverse mechanisms of immune escape ${ }^{101}$. Negative selection has also been reported prior to formation of an invasive cancer: histopathological and molecular analysis of pre-invasive lung lesions suggested that immune surveillance is more active in those that regress, relative to those that progress to invasive cancer $^{102}$.

The extent to which negative selection pressure is exerted on the developing tumour throughout its evolution is unclear: studies have suggested that neoantigen-encoding mutations may be depleted within primary tumours, indicating the impact of negative selection prior to sampling $86,91,103$. However, in a recent analysis which adjusted for single nucleotide substitution mutational signatures, no evidence of negative selection against neoantigens was found, with the exception of NSCLC ${ }^{104}$. A recent preprint reported an orthogonal approach, restricting $\mathrm{dN} / \mathrm{dS}$ to the immunopeptidome, that detected immune selection in some tumours ${ }^{105}$. Intriguingly, pre-treatment immune selection was detected at increased levels within a subset of patients with metastatic disease who responded poorly to immune checkpoint inhibitor therapy ${ }^{105}$.

Just as nonsynonymous mutations in a tumour may be immunologically functional or non-functional depending on their ability to elicit a T-cell response, so the functionality and differentiation state of T-cells may also vary between tumours. Work on NSCLC found that dysfunctional and terminally differentiated T-cells expressing PD1 and inducible T-cell costimulatory (ICOS) were more predominant in tumours with a high mutational burden, whilst progenitor-like T-cells in the early stages of differentiation expressing CD27 and CD28, and lacking signs of antigen engagement, were seen in tumours with a low mutational burden ${ }^{106}$. This is clinically significant as a gene signature of differentiation skewing was associated with a worse prognosis across multiple tumour types without immunotherapy ${ }^{106}$.

A structured immune microenvironment, manifest as spatial differences between areas of the same tumour, can be important to both the trajectory of a tumour and patient outcome. In triple-negative breast cancer, highly multiplexed imaging enabled classification of tumours by their extent of tumour-immune mixing, as either 'cold', 'mixed' or 'compartmentalized' ${ }^{107}$. Intriguingly, this feature correlated with expression of the immunotherapy targets indoleamine 2,3-dioxygenase 1 (IDO1) and PD1 ligand 1 (PDL1) on 
tumour or non-tumour cells: in the compartmentalized tumours, IDO-1 and PDL1 were expressed by non-tumour cells, such as monocytes, and these tumours were associated with improved prognosis ${ }^{107}$. Within lung adenocarcinoma, the presence of more than one 'immune-cold' region in which immune evasion might have occurred, predicted poor patient outcome irrespective of the immune phenotypes observed in the rest of the tumour ${ }^{108}$. Immune-cold regions from the same tumour were more likely to share subclonal mutations than immune-hot regions, raising the possibility that 'functional' events, which mostly remain to be elucidated, underpin the ability of an evolving tumour to evade immune rejection.

Taken together, these studies demonstrate that tumours, or parts of tumours, may have different relationships with the anti-tumour immune response and can behave differently, thereby highlighting the importance of heterogeneity in the microenvironment of a tumour towards shaping its evolution.

\section{The non-immune microenvironment and tumour evolution}

Cancers exploit local signalling networks that can profoundly impact cellular identity, to co-opt stromal cells and foster a microenvironment that is favourable to tumour growth via the angiogenic switch, epithelial-to-mesenchymal transition and other processes (reviewed elsewhere ${ }^{109}$ ). This creates regional spatial differences between parts of tumours, which may be observed by analysis of $\mathrm{pH}$ gradients, hypoxia and growth factor concentration ${ }^{110}$. Moreover, ITH may be driven in part by microenvironmental factors: this is supported by modelling data ${ }^{111}$ and implied in a study of ccRCC, where regional variations observed on imaging could not be accounted for by genetic variation ${ }^{112}$. Interactions between cancer cells and their stromal counterparts can profoundly influence the trajectory of a tumour. p53dependent senescence in hepatic stellate cells may act in a non-cell-autonomous manner to promote macrophage differentiation and an anti-tumourigenic microenvironment ${ }^{113}$. In contrast, cancer cells may co-opt the systemic environment, outside the confines of the tumour, such as in the formation of the pre-metastatic niche through tumour-secreted factors and tumour-shed vesicles ${ }^{114}$. Incoming cancer cells can then interact with this environment. For example, a co-culture system demonstrated that, in the early stages of lung metastasis, interaction between alveolar epithelial cells and disseminated breast cancer cells has been shown to influence behaviour of metastatic cells, enabling them to remain indolent and survive for long periods of time ${ }^{115}$.

Distinct clones within a tumour may also interact; clonal cooperativity within a cancer has been described in mouse models of breast cancer ${ }^{116}$. Furthermore, in a study of human colorectal cancer tissue, Schurch and colleagues used co-detection by indexing (CODEX) imaging of formalin-fixed, paraffin-embedded tissues to profile the 'cellular neighbourhoods', and found that features of their relationships, such as coupling of the immune and tumour neighbourhoods and disruption of inter-neighbourhood communication, correlated with poor prognosis ${ }^{117}$.

Understanding the extent of cooperation and 'task sharing' between cancer clones is an important area of further study. Highly multiplexed imaging modalities such as mass cytometry are increasingly facilitating the accurate phenotyping of diverse cell types obtained from tumour samples. This has given greater granularity to early observations of clonal cooperation, and relationships between cells can be described in detail ${ }^{118-120}$. Multi-omic studies that can be mapped spatially to the tumour will help to further define functional examples of tumour heterogeneity.

\section{Clinical impact of ITH}


541

542

543

544

545

546

547

548

549

550

551

552

553

554

555

556

557

558

559

560

561

562

563

564

565

566

567

568

569

570

571

572

573

574

575

576

577

578

579

580

581

582

583

584

585

586

587

588

589

590
ITH and assessments of tumour evolutionary trajectories, as well as the interplay between the cancer cell and the immune microenvironment are all important in the context of clinical questions. Will a tumour metastasise? Will it respond to therapy, and how durable will any response be? What is the prognosis of this cancer? In order to answer such questions, we must have as clear a picture as possible of the phenotype of the tumour: for this, we must comprehend the evolutionary forces that have fostered it.

\section{$\underline{\text { Metastasis }}$}

Understanding functional ITH may provide insights into the process of metastasis. The impact of cancer evolutionary dynamics on metastasis is an active area of research and our current understanding is reviewed elsewhere ${ }^{121}$.

Unpaired analysis of genomes from primary and metastatic tumours suggests that metastasis-specific genomically-encoded driver mutations are rare ${ }^{122}$. Nonetheless, the abundance of certain driver events in metastases may exceed that within the primary tumour, such as the enrichment for loss of $9 \mathrm{p}$ (containing the tumour suppressor $C D K N 2 A$ ), in metastatic ccRCC ${ }^{42}$, or mutations in $M L K 4$ (also known as MAP3K21) pan-cancer ${ }^{122}$. An enrichment within metastatic cancers for mutations of epigenetic regulators has also been characterised, adding weight to the notion that transcriptional promiscuity and phenotypic diversity may be a prerequisite for spread in some settings ${ }^{45,72,123}$. Importantly, transcriptional promiscuity need not be genomically underpinned, emphasizing the need for analysis beyond the metastatic cancer genome.

As ITH of primary tumours provides insight into their evolutionary history, so analysis of intra-tumour, and inter-tumour heterogeneity in metastases can shape our understanding of their biology. For example, in using genomics to attempt to understand the timing of metastatic dissemination, an appreciation of genomic heterogeneity within both primary tumours and metastases is essential. Under-sampling of a primary tumour may exaggerate the degree of genetic divergence between primary and metastatic lesions and hence convey metastatic divergence occurring earlier in evolutionary time, potentially contributing to a lack of consensus in the field. In a cohort of 17 patients with disseminated breast cancer, metastatic divergence was estimated to occur relatively late, at $87 \%$ of molecular time ${ }^{123}$. Conversely, a recent analysis by Curtis and colleagues, using mathematical modelling, estimated metastatic seeding to occur 2-4 years before diagnosis in colorectal, breast and lung cancers ${ }^{124}$. This study also highlighted a role for systemic anticancer treatment in promoting clonal evolution and thus influencing ITH at relapse: $57 \%$ of treated metastases showed private driver events, compared to $20 \%$ of those that were untreated. This work also serves to highlight the fact that an as-yet-undefined degree of the genetic diversity demonstrated in other studies of post-therapy tumour metastases may reflect ongoing evolution in response to treatment.

The problem with sampling also extends to questions regarding the mode of dissemination to the metastatic site. To robustly assess this, multi-region sampling of both primary and metastatic lesions is required, and there are few studies that have done this to date $^{121}$. Nonetheless, many studies have attempted to quantify the relative contributions of monoclonal (a single subclone seeds every metastasis) and polyclonal (multiple subclones seed one or more metastases) dissemination in cancer. Of note, Reiter and colleagues performed an analysis of 317 regions from 20 patients across lymph node and distant metastatic sites, to investigate the relative preponderance of metastatic seeding patterns ${ }^{125}$. They identified a higher genetic diversity among lymph node metastases than distant metastases, suggestive of polyclonal seeding patterns and more relaxed selection pressures in 
lymph node seeding clones relative to distant sites. The potential pitfalls of inferring seeding patterns from bulk sequencing is underlined by a study which presents a tool that combines inference of clonal lineage with migration histories ${ }^{126}$. By using this tool to re-analyse published sequencing data, Raphael and colleagues showed how seeding patterns might often be misclassified; for example, in a study that had originally suggested a polyclonal seeding pattern, it was posited that monoclonal seeding was in fact a more parsimonious explanation for the data ${ }^{126}$.

Whilst multi-region sampling of a resected solid primary tumour is relatively straightforward, ethical constraints prevent simultaneous sampling of multiple metastases from patients. Two strategies that may help to combat this problem and provide insights into the evolutionary history and diversity of cells within the same or different metastases are autopsy studies and circulating tumour DNA (ctDNA) analyses.

Autopsy studies allow for sampling of multiple metastases from the same patient. One such study of 76 untreated metastases from 20 patients suggested that driver mutations in this setting are typically found within all metastases and have likely occurred before metastatic dissemination ${ }^{127}$. Another analysis underlined the potential limitations of inferring phylogeny from a single metastatic sample ${ }^{128}$; this revealed pervasive branched evolution in 6 out of 7 melanoma patients and suggested that metastases in different sites may have entirely different clonal histories, as well as different active mutational processes, than the primary tumour. This is an active area of research and larger autopsy studies, such as the Posthumous Evaluation of Advanced Cancer Environment (PEACE; NCT03004755) ${ }^{129}$, may provide greater insights in the future.

Studies measuring ctDNA can help to build a picture of functional tumour heterogeneity. In a cohort of 42 patients with gastrointestinal cancers, ctDNA was demonstrated to be superior to a single metastatic biopsy at capturing mechanisms of resistance to targeted therapy in the majority of patients ${ }^{130}$. In one patient where extensive sampling was undertaken following autopsy, parallel mechanisms of resistance had evolved across different metastatic sites. This diversity could be captured in ctDNA sampling but would have been missed in any single metastatic biopsy. ctDNA collection can also help to determine the pattern and timing of metastatic spread. In a study of oesophageal adenocarcinoma, extensive sampling, including of the primary tumour, blood plasma and metastatic sites at autopsy revealed 'clonal diaspora' as the predominant mode of spread, in which multiple subclones rapidly seeded multiple metastatic sites ${ }^{131}$. In studies seeking to track pre-defined genetic events over time, targeted panel sequencing of ctDNA represents a promising avenue of research.

Immune editing is known to shape the evolution of metastasis in colorectal cancer ${ }^{101}$, and analysis of a mouse model of breast cancer metastasis by Lo and colleagues suggests the immune-microenvironment can influence the modality of dissemination ${ }^{132}$. In this study, dissemination within mice lacking natural killer (NK) cell immunity was more likely to be monoclonal, and cells that spread in clusters had lower expression of NK-activating genes, and increased expression of NK-inhibitory genes. Defining functional ITH in the context of metastasis will be a multi-faceted endeavour that will help to answer outstanding questions about its biology

\section{[H2]Informing prognosis}

The balance and degree of functional and non-functional ITH within a tumour may have important prognostic implications. ITH of driver mutations, which confer a significant fitness impact upon the cancer cell, could be considered a proxy for 'functional' subclonal diversity. Several studies have described a relationship between clonal diversity of driver 
mutations in cancer and poor outcome ${ }^{133-138}$. In pre-invasive lesions such as Barrett's esophagus, most measures of clonal genetic diversity have been found to correlate with early progression to invasive disease ${ }^{139}$. However, the relationship between ITH and outcome is nuanced. Early acquisition and clonal sweeps of driver events, resulting in a relatively homogeneous tumour, are also associated with progression to metastasis and poor outcome ${ }^{23}$, as seen in $\mathrm{ccRCC}^{42}$.

$\mathrm{CIN}$ is also a source of functional and non-functional diversity in some tumours, and gene expression correlates of CIN have long been associated with poor outcome across multiple cancer types ${ }^{61}$. In NSCLC, subclonal diversity of SCNAs was prospectively correlated with poor prognosis, whilst copy number aberrations also conferred poor survival in ccRCC $^{45,136}$. Conversely, in some settings extreme diversity may be associated with improved prognosis. For example, in some malignancies, a high mutational burden may be associated with improved patient outcomes, likely owing to increased immune surveillance ${ }^{137,140}$. Similarly, patients whose tumours have extreme levels of CIN tend to have better outcomes ${ }^{134,141,142}$. The fundamental role of CIN in tumourigenesis is underlined by the relative scarcity of SCNAs, when compared to somatic mutations, within normal human tissue ${ }^{143,144}$. Clonal expansion of mutant cells seems seldom to lead to cancer in the absence of SCNAs. A recent study of SCNAs within pre-invasive lesions also highlights their importance: shallow whole-genome sequencing in a longitudinal study of 88 patients with Barrett's esophagus demonstrated that copy-number profiles of lesions were able to predict patients' risk of progression ${ }^{145}$.

A possible consensus may emerge: ITH of driver events, in the context of tolerable amounts of CIN, is associated with poor outcomes in patients (Figure 6). This may result from ongoing subclonal selection of functional variation. However, in the presence of extreme CIN, ITH does not predict poor outcome. This paradox may relate to an inability of cells in such tumours to maintain a high-level fitness, or potentially to such tumours being exceptionally susceptible to anti-cancer therapy that may make retaining fitness impossible. A recent evolutionary model of the prognostic impact of ITH supports the view that clonal diversity, in the presence of genomic instability, is associated with faster tumour growth and reduced survival for patients ${ }^{146}$.

\section{[H2]Therapy response and resistance}

Understanding the clonal architecture of a cancer can be crucial for effective treatment. For example, clonal, rather than subclonal, neoantigens, appear to stimulate an effective immune response, and their abundance has been associated with survival in multiple cohorts treated with immune checkpoint inhibitors ${ }^{92,147}$. Also, in gastric cancers treated with an experimental fibroblast growth factor receptor (FGFR) inhibitor, response was conditional on a clonal FGFR2 amplification, and patients with a subclonal amplification failed to respond ${ }^{148}$.

Treatment resistance, either de-novo ${ }^{149}$ or pre-existing, is unfortunately a nearubiquitous feature of cancer treatment. This may arise through varied mechanisms: for example, in NSCLC, patients may develop resistance to first-generation EGFR inhibitors via the $E G F R^{\mathrm{T} 790 \mathrm{M}}$ point mutation ${ }^{150}$; in glioma, resistance to temozolamide can arise through a fusion structural variant in $M G M T^{151}$; and in acute myeloid leukaemia (AML), transcriptional plasticity may drive resistance to bromodomain and extraterminal (BET) inhibitor therapy ${ }^{152}$. An understanding of resistance mechanisms may reveal opportunities for further treatment; examples include osimertinib, which binds irreversibly to mutated EGFR-T790M, for treatment of NSCLC, or targeting of enhancer switching with lysine demethylase 1A (KDM1A) inhibition for AML treatment ${ }^{150,152}$. 
Resistance is rarely attributable to a single event; indeed, extensive CIN, promiscuous transcriptional signalling and epigenetic plasticity, all conferring unstable cellular phenotypes, may each fuel non-genetic resistance and confound multiple lines of treatment ${ }^{153-157}$. In such circumstances, targeted therapies may have limited value. Treatments that co-opt the anti-cancer immune response such as immune checkpoint blockade, adoptive T-cell therapy or vaccine therapy, however, might be less vulnerable to such resistance. Nevertheless, treatment resistance remains a significant problem ${ }^{158,159}$ and approaches to delay, or even prevent this are urgently required.

Adaptive, evolutionary-aware strategies may hold promise in improving outcomes for patients. Resistant cells can have a relative fitness disadvantage compared to their sensitive neighbours in the absence of treatment ${ }^{160-162}$, and in such a scenario, temporarily withholding treatment could cause a net growth in the sensitive population and a decline in the resistant population. Non-destructive modelling, as demonstrated in NSCLC whereby dead cells are collected from culture without disrupting the live population, suggests this approach is effective in controlling the clonal composition of tumours over time ${ }^{163}$. However, preliminary results from certain clinical trials of adaptive treatments have been disappointing ${ }^{164}$, highlighting the need for further work and a deeper understanding of the fitness costs of resistance and how this can be measured over time.

\section{[H1] Conclusions and Perspectives}

Our understanding of cancer evolution has increased exponentially in the last decade. The advent of next-generation sequencing has shed light on extensive genomic heterogeneity within cancers and given insight into the evolutionary pressures governing tumour growth.

However, it is necessary to acknowledge that further advances in this field will require not only more extensive sampling of the cancer genome across space and time, but also a more in-depth exploration of the cancer cell and its environment, moving beyond the cancer genome. Recent work, analysing the impact of structural variation, epigenomic and transcriptomic dysregulation and the immune microenvironment on cancer evolution have highlighted extensive functional variation within tumours, with profound impacts on phenotype.

Genetic and non-genetic divergence is a feature of every tumour, in part simply reflecting the random acquisition of mutations during cell division. Thus, rather than simply cataloguing diversity, future work must distinguish between functional and non-functional ITH, identifying events that might be subject to negative or positive selection during tumour evolution. Indeed, this is a fundamental issue for cancer research: notwithstanding the possibility of cure by complete surgical resection of all cancer cells present in a patient, the genetic and non-genetic trajectories of the cells within a tumour have a profound impact on disease prognosis. A thorough understanding of the relative weights exerted by various biological pulleys and levers during this process might enable us to fine-tune anti-cancer treatments and more effectively control the evolutionary fate of cancer cells.

\section{ToC blurb}

This review discusses the role of functional (impacting tumour phenotype) and nonfunctional intra-tumour heterogeneity (ITH) in cancer evolution. It highlights the importance of considering genetic and non-genetic factors and their impact on patient outcomes. 
Acknowledgements

742 The authors would like to thank Chris Bailey, Alex Frankell, Emma Colliver, CarlosMartinez Ruiz and Jonas Demeulemeester for their thoughtful review of the manuscript.

\section{Conflict of Interest}

The authors declare no conflicts of interest.

Competing interests

The authors declare no competing interests.

Author contributions

JRMB and NM both researched data for the article and made a substantial contribution to discussion of content, writing, reviewing and editing the article.

Peer review information

Nature Reviews Cancer thanks Moritz Gerstung and the other, anonymous, reviewer(s) for their contribution to the peer review of this work.

\section{Glossary:}

Chromosomal instability (CIN): A defect in which cells can gain, lose or rearrange parts of or whole chromosomes during cell division; this is a source of variation in cancer. Chromothripsis: A mutational process in which large numbers of clustered structural rearrangements occur in single or multiple chromosomes.

Molecular time: An estimate of the timing of an event, from the first cell division following fertilisation to a cell division that occurred only recently before sampling. Enhancer: A short genomic region that influences the expression of another gene in cis.

\section{$\underline{\text { References }}$}

1. Darwin, C. The origin of species by means of natural selection: or, the preservation of favored races in the struggle for life. (AL Burt New York, 1859).

2. Nowell, P. C. The clonal evolution of tumor cell populations. Science 194, 23-28 (1976).

3. Greaves, M. \& Maley, C. C. Clonal evolution in cancer. Nature 481, 306-313 (2012).

4. Williams, M. J., Werner, B., Graham, T. A. \& Sottoriva, A. Functional versus nonfunctional intratumor heterogeneity in cancer. Mol. Cell. Oncol. 3, e1162897 (2016).

5. Turajlic, S., Sottoriva, A., Graham, T. \& Swanton, C. Resolving genetic heterogeneity in cancer. Nat. Rev. Genet. 20, 404-416 (2019).

6. Marusyk, A., Janiszewska, M. \& Polyak, K. Intratumor Heterogeneity: The Rosetta

Stone of Therapy Resistance. Cancer Cell 37, 471-484 (2020).

7. McGranahan, N., Burrell, R. A., Endesfelder, D., Novelli, M. R. \& Swanton, C. Cancer chromosomal instability: therapeutic and diagnostic challenges. EMBO Rep. 13, 528-538 (2012).

8. Alexandrov, L. B. et al. Clock-like mutational processes in human somatic cells. Nat. Genet. 47, 1402-1407 (2015).

9. Coons, S. W., Johnson, P. C. \& Shapiro, J. R. Cytogenetic and Flow Cytometry DNA Analysis of Regional Heterogeneity in a Low Grade Human Glioma. Cancer Res. 55, 1569 LP - 1577 (1995).

10. Teixeira, M. R., Pandis, N., Bardi, G., Andersen, J. A. \& Heim, S. Karyotypic Comparisons of Multiple Tumorous and Macroscopically Normal Surrounding Tissue Samples from Patients with Breast Cancer. Cancer Res. 56, 855 LP - 859 (1996).

11. Navin, N. et al. Inferring tumor progression from genomic heterogeneity. Genome Res. 
20, 68-80 (2010).

12. Gerlinger, M. et al. Intratumor Heterogeneity and Branched Evolution Revealed by Multiregion Sequencing. N. Engl. J. Med. 366, 883-892 (2012).

13. OHTA, T. Slightly Deleterious Mutant Substitutions in Evolution. Nature 246, 96-98 (1973).

14. Ling, S. et al. Extremely high genetic diversity in a single tumor points to prevalence of non-Darwinian cell evolution. Proc. Natl. Acad. Sci. 112, E6496 LP-E6505 (2015).

15. Sottoriva, A. et al. A Big Bang model of human colorectal tumor growth. Nat. Genet. 47, 209-216 (2015).

16. Williams, M. J., Werner, B., Barnes, C. P., Graham, T. A. \& Sottoriva, A. Identification of neutral tumor evolution across cancer types. Nat. Genet. 48, 238-244 (2016).

17. Sun, R. et al. Between-region genetic divergence reflects the mode and tempo of tumor evolution. Nat. Genet. 49, 1015-1024 (2017).

18. Cross, W. et al. The evolutionary landscape of colorectal tumorigenesis. Nat. Ecol. Evol. 2, 1661-1672 (2018).

19. Baca, S. C. et al. Punctuated Evolution of Prostate Cancer Genomes. Cell 153, 666677 (2013).

20. Gao, R. et al. Punctuated copy number evolution and clonal stasis in triple-negative breast cancer. Nat. Genet. 48, 1119-1130 (2016).

21. Notta, F. et al. A renewed model of pancreatic cancer evolution based on genomic rearrangement patterns. Nature 538, 378-382 (2016).

22. Field, M. G. et al. Punctuated evolution of canonical genomic aberrations in uveal melanoma. Nat. Commun. 9, 116 (2018).

23. Reiter, J. G. et al. An analysis of genetic heterogeneity in untreated cancers. Nat. Rev. Cancer 639-650 (2019) doi:10.1038/s41568-019-0185-x.

24. Papaemmanuil, E. et al. Clinical and biological implications of driver mutations in myelodysplastic syndromes. Blood 122, 3616-27; quiz 3699 (2013).

25. Brastianos, P. K. et al. Genomic Characterization of Brain Metastases Reveals Branched Evolution and Potential Therapeutic Targets. Cancer Discov. 5, 1164-1177 (2015).

26. Landau, D. A. et al. Mutations driving CLL and their evolution in progression and relapse. Nature 526, 525-530 (2015).

27. Yates, L. R. et al. Subclonal diversification of primary breast cancer revealed by multiregion sequencing. Nat. Med. 21, 751-759 (2015).

28. Mitchell, T. J. et al. Timing the Landmark Events in the Evolution of Clear Cell Renal Cell Cancer: TRACERx Renal. Cell 173, 611-623.e17 (2018).

29. Gerstung, M. et al. The evolutionary history of 2,658 cancers. Nature 578, (2020).

30. Nik-Zainal, S. et al. The life history of 21 breast cancers. Cell 149, 994-1007 (2012).

31. Kessler, D. A. \& Levine, H. Large population solution of the stochastic LuriaDelbrück evolution model. Proc. Natl. Acad. Sci. 110, 11682-11687 (2013).

32. Caravagna, G. et al. Subclonal reconstruction of tumors by using machine learning and population genetics. Nat. Genet. 52, 898-907 (2020).

33. Litchfield, K. et al. Representative Sequencing: Unbiased Sampling of Solid Tumor Tissue. Cell Rep. 31, 107550 (2020).

34. Durinck, S. et al. Temporal dissection of tumorigenesis in primary cancers. Cancer Discov. 1, 137-143 (2011).

35. Martincorena, I. et al. High burden and pervasive positive selection of somatic mutations in normal human skin. Science (80-. ). 348, 880-886 (2015).

36. Martincorena, I. et al. Universal Patterns of Selection in Cancer and Somatic Tissues. 
Cell 171, 1029-1041.e21 (2017).

37. McGranahan, N. \& Swanton, C. Clonal Heterogeneity and Tumor Evolution: Past, Present, and the Future. Cell 168, 613-628 (2017).

38. Tarabichi, M. et al. Neutral tumor evolution? Nat. Genet. 50, 1630-1633 (2018).

39. Nam, A. S., Chaligne, R. \& Landau, D. A. Integrating genetic and non-genetic determinants of cancer evolution by single-cell multi-omics. Nat. Rev. Genet. (2020) doi:10.1038/s41576-020-0265-5.

40. Laks, E. et al. Clonal Decomposition and DNA Replication States Defined by Scaled Single-Cell Genome Sequencing. Cell 179, 1207-1221.e22 (2019).

41. Cross, W. et al. Stabilising selection causes grossly altered but stable karyotypes in metastatic colorectal cancer. bioRxiv 2020.03.26.007138 (2020) doi:10.1101/2020.03.26.007138.

42. Turajlic, S. et al. Tracking Cancer Evolution Reveals Constrained Routes to Metastases: TRACERx Renal. Cell 173, 581-594.e12 (2018).

43. Gundem, G. et al. The evolutionary history of lethal metastatic prostate cancer. Nature 520, 353-357 (2015).

44. Suzuki, H. et al. Mutational landscape and clonal architecture in grade II and III gliomas. Nat. Genet. 47, 458-468 (2015).

45. Jamal-Hanjani, M. et al. Tracking the evolution of non-small-cell lung cancer. N. Engl. J. Med. 376, 2109-2121 (2017).

46. Watkins, T. B. K. et al. Pervasive chromosomal instability and karyotype order in tumour evolution. Nature (2020) doi:10.1038/s41586-020-2698-6.

47. Bolhaqueiro, A. C. F. et al. Ongoing chromosomal instability and karyotype evolution in human colorectal cancer organoids. Nat. Genet. 51, 824-834 (2019).

48. Flavahan, W. A., Gaskell, E. \& Bernstein, B. E. Epigenetic plasticity and the hallmarks of cancer. Science (80-. ). 357, (2017).

49. Clarke, T. L. et al. Histone Lysine Methylation Dynamics Control EGFR DNA CopyNumber Amplification. Cancer Discov. 10, 306-325 (2020).

50. Chen, H. et al. A Pan-Cancer Analysis of Enhancer Expression in Nearly 9000 Patient Samples. Cell 173, 386-399.e12 (2018).

51. Gröbner, S. N. et al. The landscape of genomic alterations across childhood cancers. Nature 555, 321-327 (2018).

52. Kahles, A. et al. Comprehensive Analysis of Alternative Splicing Across Tumors from 8,705 Patients. Cancer Cell 34, 211-224.e6 (2018).

53. Shiraishi, Y. et al. A comprehensive characterization of cis-acting splicing-associated variants in human cancer. Genome Res. 28, 1111-1125 (2018).

54. Demircioğlu, D. et al. A Pan-cancer Transcriptome Analysis Reveals Pervasive Regulation through Alternative Promoters. Cell 178, 1465-1477.e17 (2019).

55. Calabrese, C. et al. Genomic basis for RNA alterations in cancer. Nature 578, 129-136 (2020).

56. Zhang, Y. et al. High-coverage whole-genome analysis of 1220 cancers reveals hundreds of genes deregulated by rearrangement-mediated cis-regulatory alterations. Nat. Commun. 11, 736 (2020).

57. Bailey, M. H. et al. Comprehensive Characterization of Cancer Driver Genes and Mutations. Cell 173, 371-385.e18 (2018).

58. Sondka, Z. et al. The COSMIC Cancer Gene Census: describing genetic dysfunction across all human cancers. Nat. Rev. Cancer 18, 696-705 (2018).

59. Baysal, B. E., Sharma, S., Hashemikhabir, S. \& Janga, S. C. RNA Editing in Pathogenesis of Cancer. Cancer Res. 77, 3733-3739 (2017).

60. Chen, L. et al. Recoding RNA editing of AZIN1 predisposes to hepatocellular 
908

909

910

911

912

913

914

915

916

917

918

919

920

921

922

923

924

925

926

927

928

929

930

931

932

933

934

935

936

937

938

939

940

carcinoma. Nat. Med. 19, 209-216 (2013).

61. Carter, S. L., Eklund, A. C., Kohane, I. S., Harris, L. N. \& Szallasi, Z. A signature of chromosomal instability inferred from gene expression profiles predicts clinical outcome in multiple human cancers. Nat. Genet. 38, 1043-1048 (2006).

62. Biswas, D. et al. A clonal expression biomarker associates with lung cancer mortality. Nat. Med. 25, 1540-1548 (2019).

63. Salami, S. S., Tomlins, S. A. \& Palapattu, G. S. Transcriptomic Heterogeneity in Multifocal Prostate Cancer. JCI 201, 666-667 (2019).

64. Thomsen, M. B. H. et al. Comprehensive multiregional analysis of molecular heterogeneity in bladder cancer. Sci. Rep. 7, 11702 (2017).

65. Aran, D., Sirota, M. \& Butte, A. J. Systematic pan-cancer analysis of tumour purity. Nat. Commun. 6, 8971 (2015).

66. Wang, F. et al. Integrated transcriptomic-genomic tool Texomer profiles cancer tissues. Nat. Methods 16, 401-404 (2019).

67. Teschendorff, A. E. \& Enver, T. Single-cell entropy for accurate estimation of differentiation potency from a cell's transcriptome. Nat. Commun. 8, 15599 (2017).

68. Marjanovic, N. D. et al. Emergence of a High-Plasticity Cell State during Lung Cancer Evolution. Cancer Cell (2020) doi:https://doi.org/10.1016/j.ccell.2020.06.012.

69. Lafave, L. M. et al. Epigenomic state transitions characterize tumor progression in lung adenocarcinoma. Cancer Cell 212-228 (2020) doi:10.1016/j.ccell.2020.06.006.

70. Izzo, F. et al. DNA methylation disruption reshapes the hematopoietic differentiation landscape. Nat. Genet. 52, 378-387 (2020).

71. Raphael, B. J. Integrated Genomic Characterization of Pancreatic Ductal Adenocarcinoma. Cancer Cell 32, 185-203.e13 (2017).

72. McDonald, O. G. et al. Epigenomic reprogramming during pancreatic cancer progression links anabolic glucose metabolism to distant metastasis. Nat. Genet. 49, 367-376 (2017).

73. Pastore, A. et al. Corrupted coordination of epigenetic modifications leads to diverging chromatin states and transcriptional heterogeneity in CLL. Nat. Commun. 10, 1874 (2019).

74. Hua, X. et al. Genetic and epigenetic intratumor heterogeneity impacts prognosis of lung adenocarcinoma. Nat. Commun. 2459 (2020) doi:10.1038/s41467-020-16295-5.

75. Zhu, B. et al. The genomic and epigenomic evolutionary history of papillary renal cell carcinomas. Nat. Commun. 11, 3096 (2020).

76. Mertins, P. et al. Proteogenomics connects somatic mutations to signalling in breast cancer. Nature 534, 55-62 (2016).

77. Zhang, H. et al. Integrated Proteogenomic Characterization of Human High-Grade Serous Ovarian Cancer. Cell 166, 755-765 (2016).

78. Mun, D.-G. et al. Proteogenomic Characterization of Human Early-Onset Gastric Cancer. Cancer Cell 35, 111-124.e10 (2019).

79. Chen, Y. J. et al. Proteogenomics of Non-smoking Lung Cancer in East Asia Delineates Molecular Signatures of Pathogenesis and Progression. Cell 182, 226244.e17 (2020).

80. Gillette, M. A. et al. Proteogenomic Characterization Reveals Therapeutic Vulnerabilities in Lung Adenocarcinoma. Cell 182, 200-225.e35 (2020).

81. Xu, J.-Y. et al. Integrative Proteomic Characterization of Human Lung Adenocarcinoma. Cell 182, 245-261.e17 (2020).

82. Hanahan, D. \& Weinberg, R. A. Hallmarks of Cancer: The Next Generation. Cell 144, 646-674 (2011).

83. Marty, R. et al. MHC-I Genotype Restricts the Oncogenic Mutational Landscape. Cell 
171, 1272-1283.e15 (2017).

84. Marty Pyke, R. et al. Evolutionary Pressure against MHC Class II Binding Cancer Mutations. Cell 175, 416-428.e13 (2018).

85. DuPage, M., Mazumdar, C., Schmidt, L. M., Cheung, A. F. \& Jacks, T. Expression of tumour-specific antigens underlies cancer immunoediting. Nature 482, 405-409 (2012).

86. Rooney, M. S., Shukla, S. A., Wu, C. J., Getz, G. \& Hacohen, N. Molecular and Genetic Properties of Tumors Associated with Local Immune Cytolytic Activity. Cell 160, 48-61 (2015).

87. McGranahan, N. et al. Allele-Specific HLA Loss and Immune Escape in Lung Cancer Evolution. Cell 171, 1259-1271.e11 (2017).

88. Anagnostou, V. et al. Evolution of neoantigen landscape during immune checkpoint blockade in non-small cell lung cancer. Cancer Discov. 7, 264-276 (2017).

89. Rosenthal, R. et al. Neoantigen-directed immune escape in lung cancer evolution. Nature 567, 479-485 (2019).

90. Jiménez-Sánchez, A. et al. Unraveling tumor-immune heterogeneity in advanced ovarian cancer uncovers immunogenic effect of chemotherapy. Nat. Genet. 52, 582593 (2020).

91. Davoli, T., Uno, H., Wooten, E. C. \& Elledge, S. J. Tumor aneuploidy correlates with markers of immune evasion and with reduced response to immunotherapy. Science (80-. ). 355, (2017).

92. McGranahan, N. et al. Clonal neoantigens elicit T cell immunoreactivity and sensitivity to immune checkpoint blockade. Science (80-. ). 351, 1463-1470 (2016).

93. Joshi, K. et al. Spatial heterogeneity of the T cell receptor repertoire reflects the mutational landscape in lung cancer. Nat. Med. 25, 1549-1559 (2019).

94. Roh, W. et al. Integrated molecular analysis of tumor biopsies on sequential CTLA-4 and PD-1 blockade reveals markers of response and resistance. Sci. Transl. Med. 9, eaah3560 (2017).

95. Tumeh, P. C. et al. PD-1 blockade induces responses by inhibiting adaptive immune resistance. Nature 515, 568-571 (2014).

96. Snyder, A. et al. Contribution of systemic and somatic factors to clinical response and resistance to PD-L1 blockade in urothelial cancer: An exploratory multi-omic analysis. PLOS Med. 14, e1002309 (2017).

97. Wolf, Y. et al. UVB-Induced Tumor Heterogeneity Diminishes Immune Response in Melanoma. Cell 179, 219-235.e21 (2019).

98. Gejman, R. S. et al. Rejection of immunogenic tumor clones is limited by clonal fraction. Elife 7, e41090 (2018).

99. Maire, C. L. et al. Glioma escape signature and clonal development under immune pressure. J. Clin. Invest. 130, 5257-5271 (2020).

100. Zhang, A. W. et al. Interfaces of Malignant and Immunologic Clonal Dynamics in Ovarian Cancer. Cell 173, 1755-1769.e22 (2018).

101. Angelova, M. et al. Evolution of Metastases in Space and Time under Immune Selection. Cell 175, 751-765.e16 (2018).

102. Pennycuick, A. et al. Immune surveillance in clinical regression of pre-invasive squamous cell lung cancer. Cancer Discov. CD-19-1366 (2020) doi:10.1158/21598290.CD-19-1366.

103. Zapata, L. et al. Negative selection in tumor genome evolution acts on essential cellular functions and the immunopeptidome. Genome Biol. 19, 67 (2018).

104. Eynden, J. Van Den, Jiménez-sánchez, A., Miller, M. L. \& Larsson, E. Lack of detectable neoantigen depletion signals in the untreated cancer genome. Nat. Genet. 
(2019) doi:10.1038/s41588-019-0532-6.

105. Zapata, L. et al. $\mathrm{dN} / \mathrm{dS}$ dynamics quantify tumour immunogenicity and predict response to immunotherapy. bioRxiv 2020.07.21.215038 (2020) doi:10.1101/2020.07.21.215038.

106. Ghorani, E. et al. The T cell differentiation landscape is shaped by tumour mutations in lung cancer. Nat. Cancer 1, 546-561 (2020).

107. Keren, L. et al. A Structured Tumor-Immune Microenvironment in Triple Negative Breast Cancer Revealed by Multiplexed Ion Beam Imaging. Cell 174, 1373-1387.e19 (2018).

108. Abduljabbar, K. et al. Geospatial immune variability illuminates differential evolution of lung adenocarcinoma. Nat. Med. 26, 1054-1062 (2020).

109. Wang, M. et al. Role of tumor microenvironment in tumorigenesis. J. Cancer 8, 761773 (2017).

110. Korenchan, D. E. \& Flavell, R. R. Spatiotemporal pH Heterogeneity as a Promoter of Cancer Progression and Therapeutic Resistance. Cancers (Basel). 11, 1026 (2019).

111. Lloyd, M. C. et al. Darwinian Dynamics of Intratumoral Heterogeneity: Not Solely Random Mutations but Also Variable Environmental Selection Forces. Cancer Res. 76, 3136-3144 (2016).

112. Hoefflin, R. et al. Spatial niche formation but not malignant progression is a driving force for intratumoural heterogeneity. Nat. Commun. 7, 11845 (2016).

113. Lujambio, A. et al. Non-Cell-Autonomous Tumor Suppression by p53. Cell 153, 449460 (2013).

114. Peinado, H. et al. Pre-metastatic niches: organ-specific homes for metastases. Nat. Rev. Cancer 17, 302-317 (2017).

115. Montagner, M. et al. Crosstalk with lung epithelial cells regulates Sfrp2-mediated latency in breast cancer dissemination. Nat. Cell Biol. 22, 289-296 (2020).

116. Marusyk, A. et al. Non-cell-autonomous driving of tumour growth supports sub-clonal heterogeneity. Nature 514, 54-58 (2014).

117. Schürch, C. M. et al. Coordinated Cellular Neighborhoods Orchestrate Antitumoral Immunity at the Colorectal Cancer Invasive Front. Cell 182, 1341-1359.e19 (2020).

118. Wagner, J. et al. A Single-Cell Atlas of the Tumor and Immune Ecosystem of Human Breast Cancer. Cell 177, 1330-1345.e18 (2019).

119. Ali, H. R. et al. Imaging mass cytometry and multiplatform genomics define the phenogenomic landscape of breast cancer. Nat. Cancer 1, 163-175 (2020).

120. Jackson, H. W. et al. The single-cell pathology landscape of breast cancer. Nature $\mathbf{5 7 8}$, 615-620 (2020).

121. Birkbak, N. J. \& McGranahan, N. Cancer Genome Evolutionary Trajectories in Metastasis. Cancer Cell 37, 8-19 (2020).

122. Priestley, P. et al. Pan-cancer whole-genome analyses of metastatic solid tumours. Nature 575, 210-216 (2019).

123. Yates, L. R. et al. Genomic Evolution of Breast Cancer Metastasis and Relapse. Cancer Cell 32, 169-184.e7 (2017).

124. Hu, Z., Li, Z., Ma, Z. \& Curtis, C. Multi-cancer analysis of clonality and the timing of systemic spread in paired primary tumors and metastases. Nat. Genet. 52, 701-708 (2020).

125. Reiter, J. G. et al. Lymph node metastases develop through a wider evolutionary bottleneck than distant metastases. Nat. Genet. 52, 692-700 (2020).

126. El-Kebir, M., Satas, G. \& Raphael, B. J. Inferring parsimonious migration histories for metastatic cancers. Nat. Genet. 50, 718-726 (2018).

127. Reiter, J. G. et al. Minimal functional driver gene heterogeneity among untreated 
metastases. Science 361, 1033-1037 (2018).

128. Rabbie, R. et al. Multi-site clonality analysis uncovers pervasive heterogeneity across melanoma metastases. Nat. Commun. 11, 4306 (2020).

129. NCT03004755. The PEACE (Posthumous Evaluation of Advanced Cancer Environment) Study (PEACE). ClinicalTrials.gov https://clinicaltrials.gov/ct2/show/NCT03004755.

130. Parikh, A. R. et al. Liquid versus tissue biopsy for detecting acquired resistance and tumor heterogeneity in gastrointestinal cancers. Nat. Med. 25, 1415-1421 (2019).

131. Noorani, A. et al. Genomic evidence supports a clonal diaspora model for metastases of esophageal adenocarcinoma. Nat. Genet. 52, 74-83 (2020).

132. Lo, H. C. et al. Resistance to natural killer cell immunosurveillance confers a selective advantage to polyclonal metastasis. Nat. Cancer (2020) doi:10.1038/s43018-0200068-9.

133. Landau, D. A. et al. Evolution and Impact of Subclonal Mutations in Chronic Lymphocytic Leukemia. Cell 152, 714-726 (2013).

134. Andor, N. et al. Pan-cancer analysis of the extent and consequences of intratumor heterogeneity. Nat. Med. 22, 105-113 (2016).

135. Rye, I. H. et al. Intratumor heterogeneity defines treatment-resistant HER2+ breast tumors. Mol. Oncol. 12, 1838-1855 (2018).

136. Turajlic, S. et al. Deterministic Evolutionary Trajectories Influence Primary Tumor Growth: TRACERx Renal. Cell 173, 595-610.e11 (2018).

137. Cerrano, M. et al. Prognostic Impact of Clonal Diversity in Acute Myeloid Leukemia (AML) Treated with Intensive Chemotherapy (IC). Blood 134, 2700 (2019).

138. Iacobuzio-Donahue, C. A., Litchfield, K. \& Swanton, C. Intratumor heterogeneity reflects clinical disease course. Nat. Cancer 1, 3-6 (2020).

139. Martinez, P. et al. Dynamic clonal equilibrium and predetermined cancer risk in Barrett's oesophagus. Nat. Commun. 7, 12158 (2016).

140. Wei, S. C., Duffy, C. R. \& Allison, J. P. Fundamental Mechanisms of Immune Checkpoint Blockade Therapy. Cancer Discov. 8, 1069-1086 (2018).

141. Birkbak, N. J. et al. Paradoxical relationship between chromosomal instability and survival outcome in cancer. Cancer Res. 71, 3447-3452 (2011).

142. Jamal-Hanjani, M. et al. Extreme chromosomal instability forecasts improved outcome in ER-negative breast cancer: a prospective validation cohort study from the TACT trial. Ann. Oncol. Off. J. Eur. Soc. Med. Oncol. 26, 1340-1346 (2015).

143. Lawson, A. R. J. et al. Extensive heterogeneity in somatic mutation and selection in the human bladder. Science (80-. ). 370, 75-82 (2020).

144. Moore, L. et al. The mutational landscape of normal human endometrial epithelium. Nature 580, 640-646 (2020).

145. Killcoyne, S. et al. Genomic copy number predicts esophageal cancer years before transformation. Nat. Med. (2020) doi:10.1038/s41591-020-1033-y.

146. Noble, R. \& Burley, J. T. When, why and how tumour clonal diversity predicts survival. Evol. Appl. 1-11 (2020) doi:10.1111/eva.13057.

147. Riaz, N. et al. Tumor and Microenvironment Evolution during Immunotherapy with Nivolumab. Cell 171, 934-949.e16 (2017).

148. Pearson, A. et al. High-Level Clonal FGFR Amplification and Response to FGFR Inhibition in a Translational Clinical Trial. Cancer Discov. 6, 838-851 (2016).

149. Russo, M. et al. Adaptive mutability of colorectal cancers in response to targeted therapies. Science (80-. ). 366, 1473-1480 (2019).

150. Mok, T. S. et al. Osimertinib or platinum-pemetrexed in EGFR T790M-Positive lung cancer. N. Engl. J. Med. 376, 629-640 (2017). 
151. Oldrini, B. et al. MGMT genomic rearrangements contribute to chemotherapy resistance in gliomas. Nat. Commun. 11, 3883 (2020).

152. Bell, C. C. et al. Targeting enhancer switching overcomes non-genetic drug resistance in acute myeloid leukaemia. Nat. Commun. 10, 2723 (2019).

153. Fong, C. Y. et al. BET inhibitor resistance emerges from leukaemia stem cells. Nature 525, 538-542 (2015).

154. Hinohara, K. et al. KDM5 Histone Demethylase Activity Links Cellular Transcriptomic Heterogeneity to Therapeutic Resistance. Cancer Cell 34, 939-953.e9 (2018).

155. McGranahan, N. \& Swanton, C. Neoantigen quality, not quantity. Sci. Transl. Med. 11, eaax7918 (2019).

156. Rambow, F. et al. Toward Minimal Residual Disease-Directed Therapy in Melanoma. Cell 174, 843-855.e19 (2018).

157. Pleasance, E. et al. Pan-cancer analysis of advanced patient tumors reveals interactions between therapy and genomic landscapes. Nat. Cancer 1, 452-468 (2020).

158. Gao, J. et al. Loss of IFN- $\gamma$ Pathway Genes in Tumor Cells as a Mechanism of Resistance to Anti-CTLA-4 Therapy. Cell 167, 397-404.e9 (2016).

159. Sahin, U. et al. Personalized RNA mutanome vaccines mobilize poly-specific therapeutic immunity against cancer. Nature 547, 222-226 (2017).

160. Siravegna, G. et al. Clonal evolution and resistance to EGFR blockade in the blood of colorectal cancer patients. Nat. Med. 21, 795-801 (2015).

161. Xue, Y. et al. An approach to suppress the evolution of resistance in BRAF(V600E)mutant cancer. Nat. Med. 23, 929-937 (2017).

162. Gopal, P. et al. Clonal selection confers distinct evolutionary trajectories in BRAFdriven cancers. Nat. Commun. 10, 5143 (2019).

163. Acar, A. et al. Exploiting evolutionary steering to induce collateral drug sensitivity in cancer. Nat. Commun. 11, 1923 (2020).

164. Algazi, A. et al. Abstract CT013: SWOG S1320: Improved progression-free survival with continuous compared to intermittent dosing with dabrafenib and trametinib in patients with BRAF mutated melanoma. Cancer Res. 80, CT013 LP-CT013 (2020).

Highlighted Refs (not included in the actual References section because Mendeley deletes them)

1. Williams \#15

a. This study demonstrates that a subset of tumours evolve without clear evidence of subclonal selection

2. Gerstung \#28

a. As part of the Pan Cancer Analysis of Whole Genomes, this study provides an overview of evolutionary patterns across cancer types, identifying different driver events that typically occur early or late in cancer.

3. Watkins \#46

a. This multi-region, pan cancer analysis of tumour karyotype uncovers parallel evolution of events within different subclones in one third of tumours sampled, and identifies the important role of chromosomal instability in generating subclonal diversity in cancer.

4. Calabrese \#56

a. This pan-cancer study of paired whole genomes and transcriptomes illustrates the variety of transcriptomic alterations in cancer, and underlines the influence of copy number events and non-coding mutations on gene expression.

5. Marjanovic \#67 
a. This study of a mouse model of lung adenocarcinoma illustrates that highly

6. McDonald \#70 plastic stem-like cells with diverse transcriptional states drive resistance to therapy and poor clinical outcome.

a. This study of pancreatic cancer is an example of the important potential role of non-genetic variation in cancer evolution.

\section{Rosenthal \#87}

1152

1153

a. This work highlights the role of immune editing in shaping early cancer evolution by negative selection, as well as the diversity of mechanisms of immune evasion.

8. Wolf \#95

a. Here, a mouse model of melanoma is used to illustrate that increased clonal diversity of a developing tumour is associated with evasion of the anti-cancer immune response

9. Rambow \#154

a. This work highlights the role played by stem-like cancer cells in non-genetic mechanisms of resistance to cancer therapy. 
Figure 1: Functional and non-functional intra-tumour heterogeneity in tumour evolution

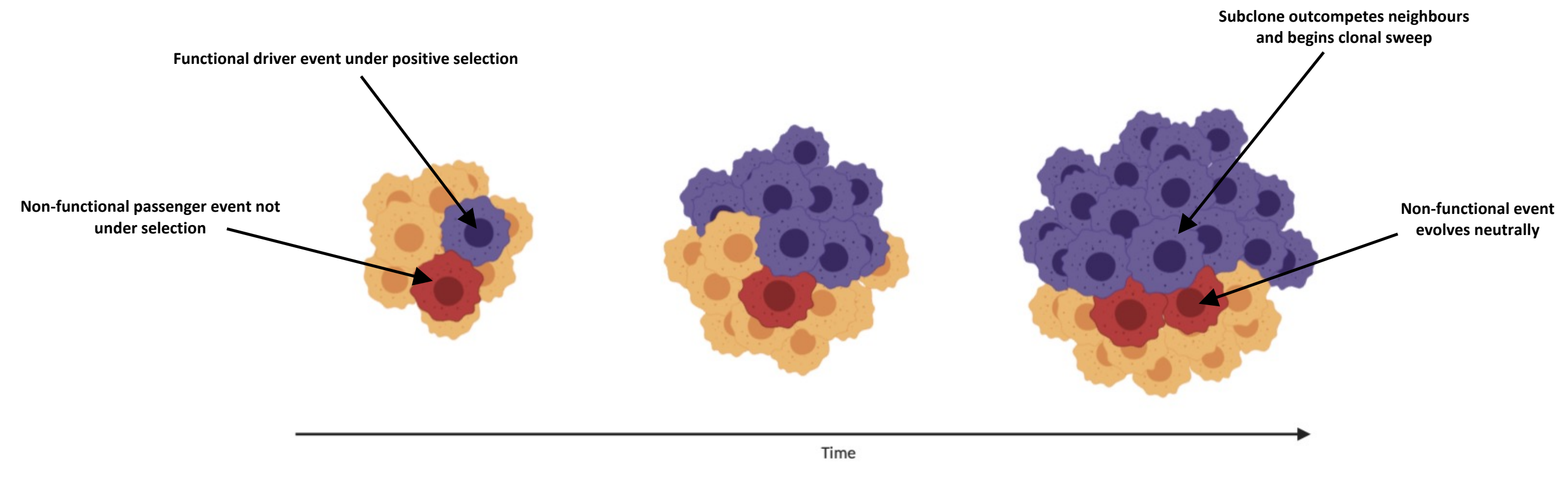

Fig. 1 | Functional and non-functional intra-tumour heterogeneity in tumour evolution. The increased rate of phenotypic variation in cancers compared with normal tissues means that new subclones arise and compete. A minority contain a driver event, such as a genetic mutation or copy number alteration, that grants a selective advantage. These subclones may grow at a faster rate than their neighbours and outcompete them in a 'selective sweep'. 
Figure 2: Methods of assessing tumour evolution: clonal frequency inference

\section{Actual tumour evolution}

i)

size

ii)

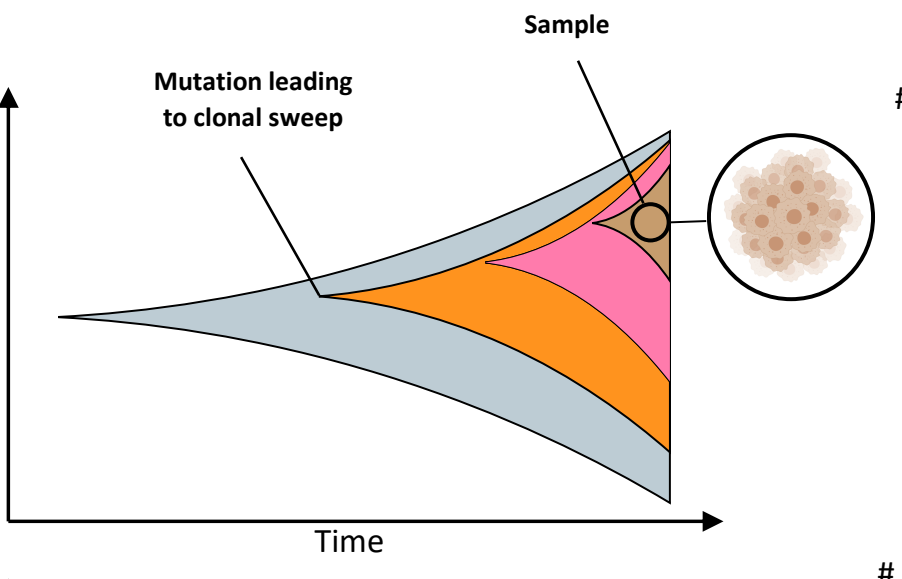

Clonal inference from variant allele frequency (VAF)

\# mutations

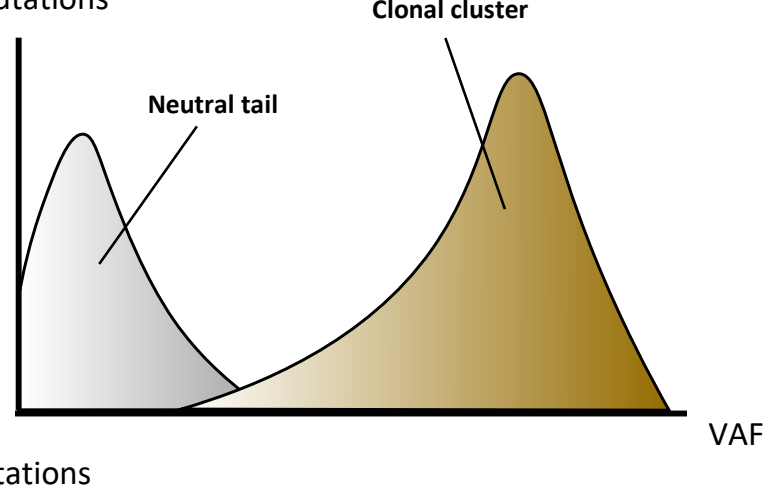

\# mutations

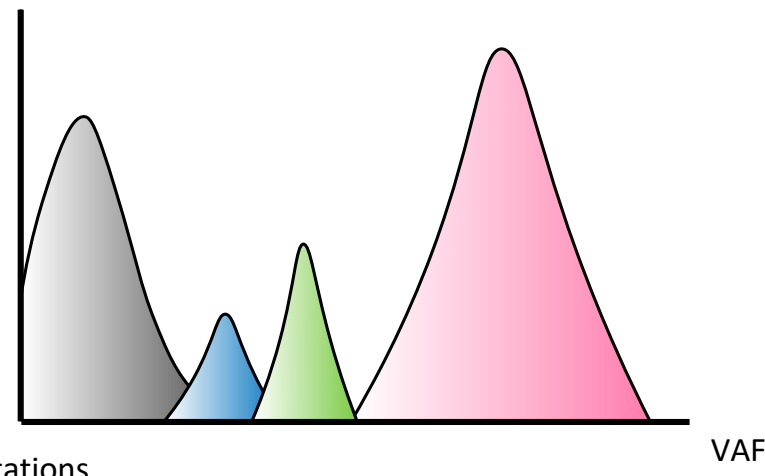

\# mutations iii)

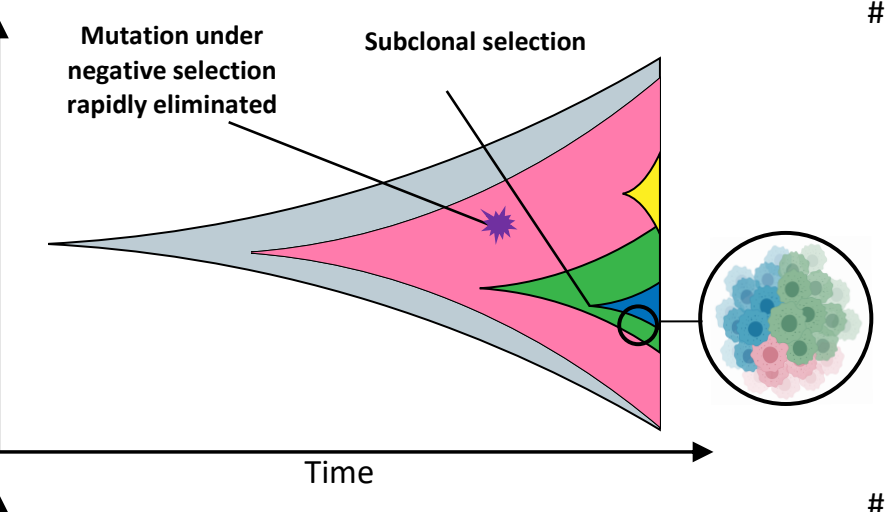

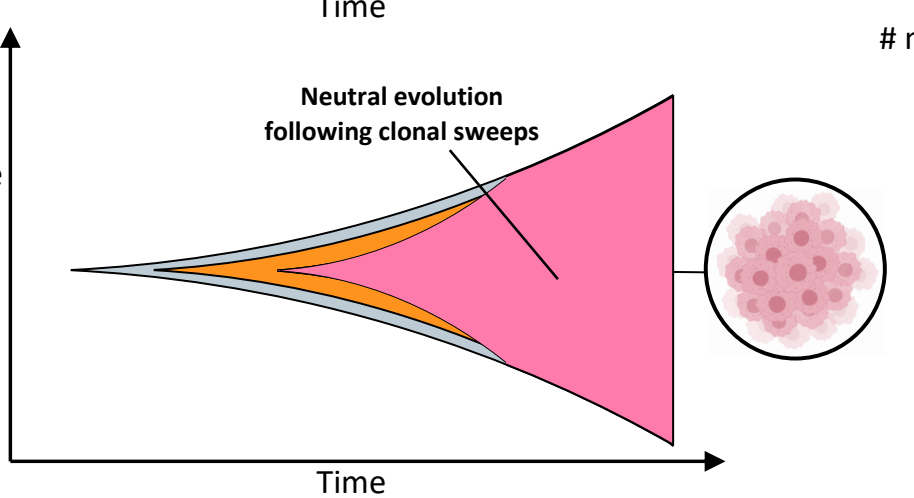

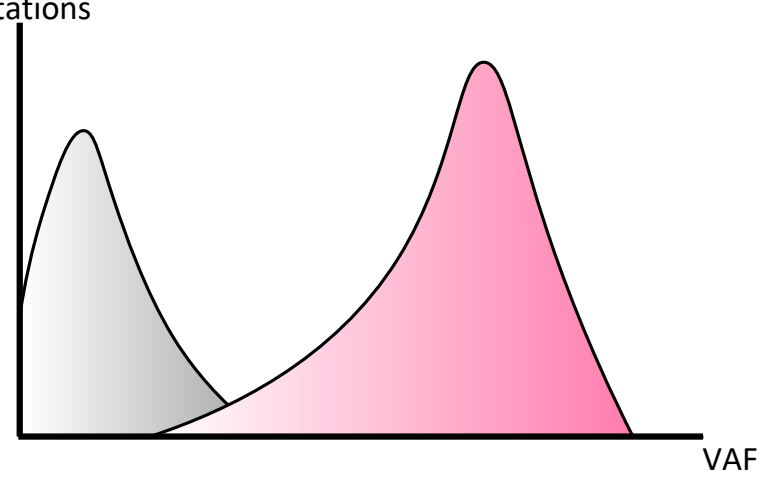

Inferred tumour evolution

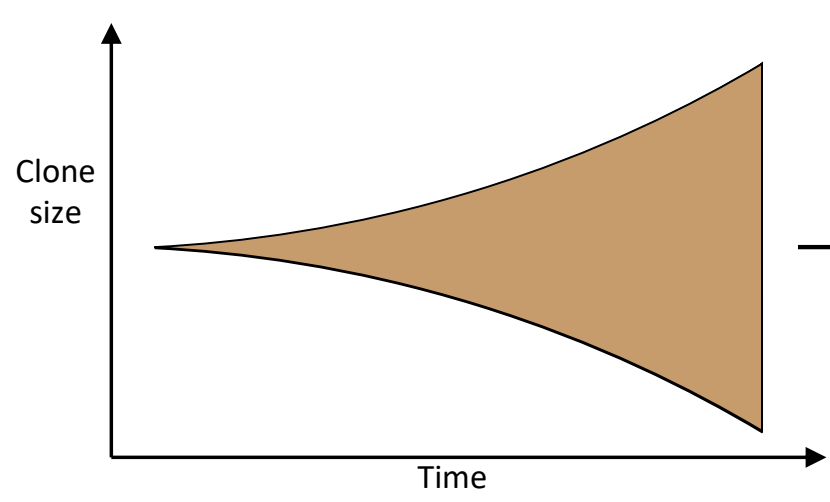

Unable to identify multiple separate selective sweeps, and so subclonal selection missed

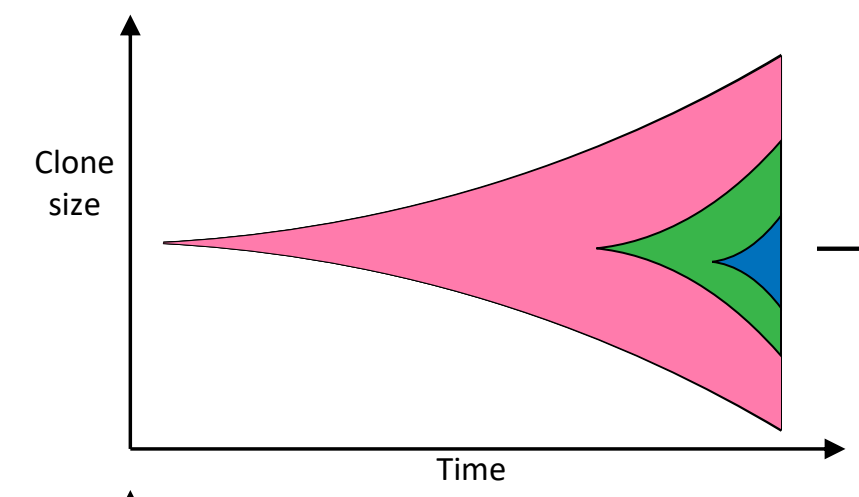

Identifies subclonal selection, but sampling misses minor subclone. Previous negative selection missed.

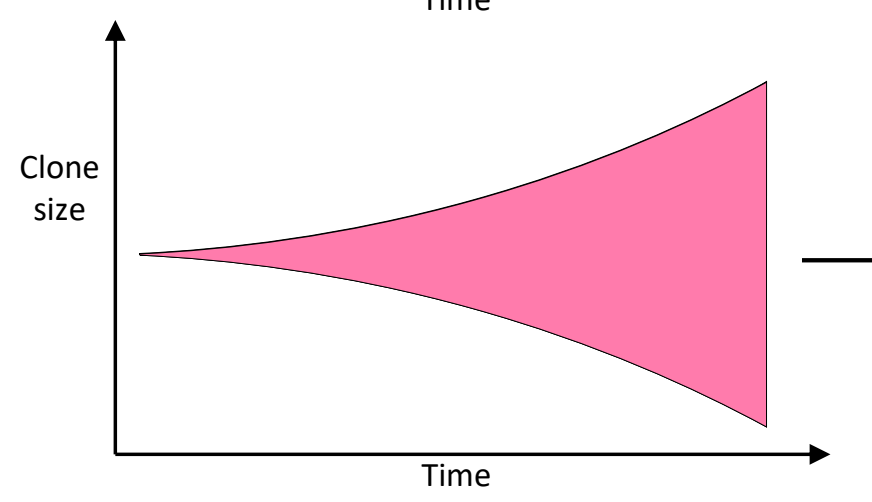

Correctly identifies neutral olution following early selective sweep. 
Fig. 2 I Methods of assessing tumour evolution and clonal frequency

inference. Three examples of tumour evolution (left panels), with inferred

clonal variant allele frequencies (VAFs) (middle panels) and tumour

evolution (right panels). Clusters of mutations are ordered according to

VAFs; the clonal cluster contains many high-VAF mutations, whereas the 'neutral tail' (coloured grey) contains mutations with lower VAFs.

Interpretation can be confounded by sampling. a I Ongoing subclonal selection leads to formation of subclones (blue/grey, orange and blue).

All blue/grey, orange and blue mutations appear clonal within the sample, and so have indistinguishable VAFs. Previous selective sweeps are not

distinguishable, and so subclonal selection may not be identified.

b I Mutations (orange, green and purple) have different VAFs, so subclones

are identifiable. Previous negative selection in this tumour is not identified.

c I Tumour evolution is reconstructed relatively accurately, with a previous

clonal sweep and neutral ongoing evolution, although previous clonal

sweeps that occurred very early are not distinguishable. 
Positive selection
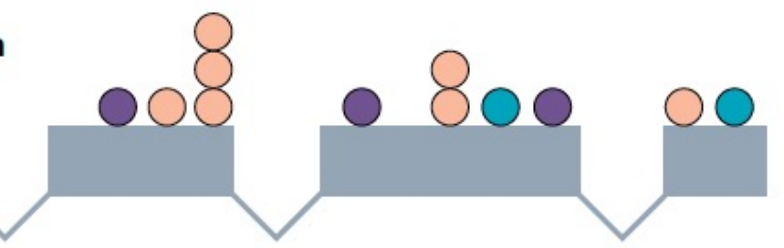

Neutral evolution
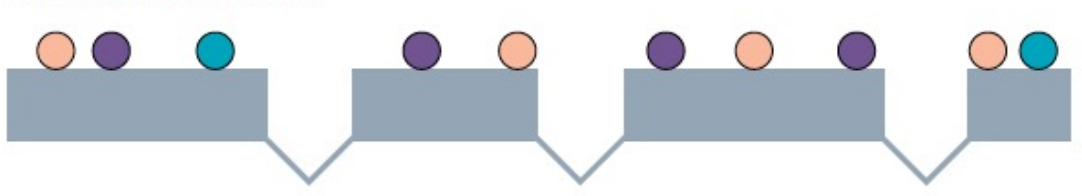

Negative selection
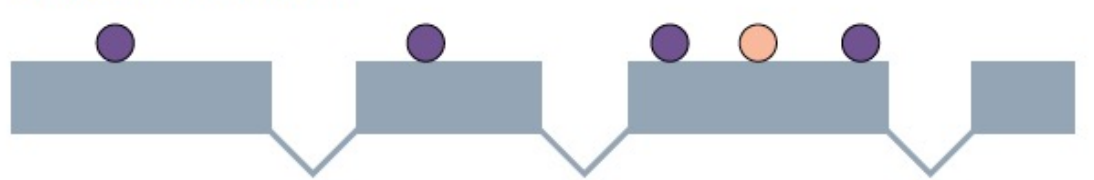

b Example 'cohort' of heterogeneous tumours

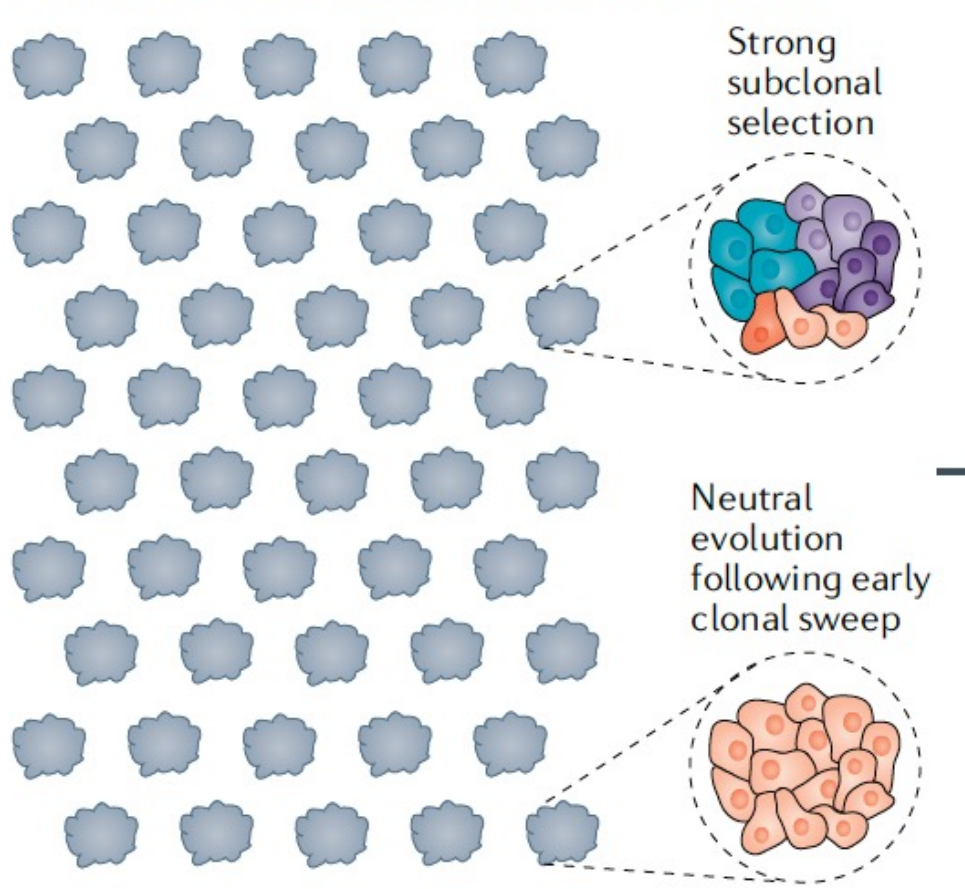

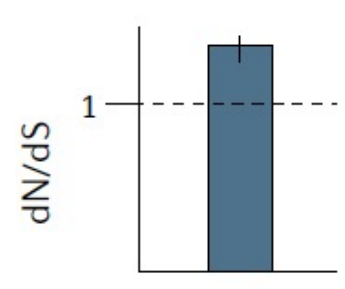
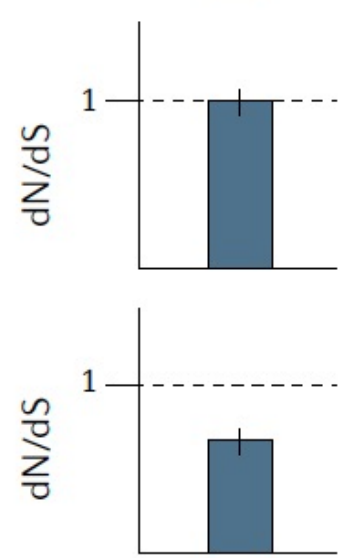

Missense mutation

Truncating mutation

Synonymous mutation

c $\mathrm{dN} / \mathrm{dS}$ within the example cohort

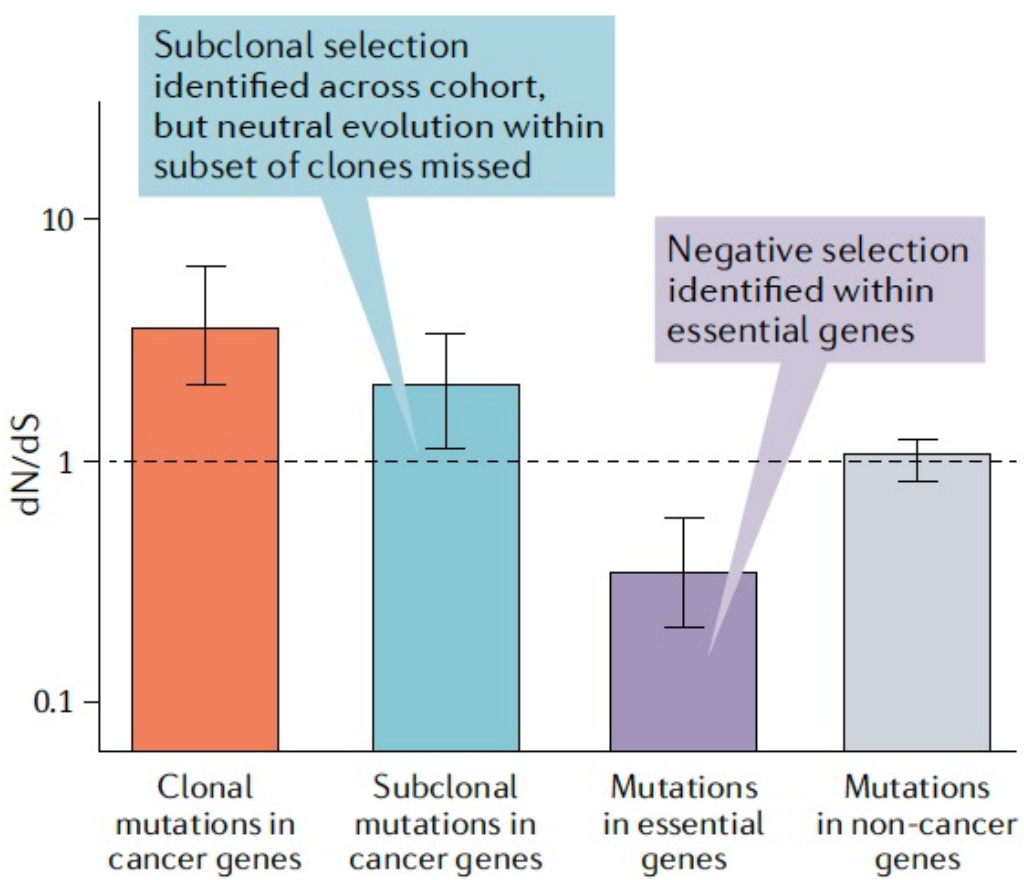

Fig. 3 I Methods of assessing tumour

evolution: dN/dS. a I This method uses the ratio

of non- synonymous mutations

at non- synonymous sites to synonymous

mutations at synonymous sites to infer selection.

Ratio of substitution rates at

non- synonymous sites versus synonymous sites $(\mathrm{dN} / \mathrm{dS})>1$ indicates that the rate of non-

synonymous mutations is above

the expected background, and signifies positive selection within a given gene or locus. $d N / d S=1$ suggests mutations

in that gene are neutral. $\mathrm{dN} / \mathrm{dS}<1$ suggests negative or purifying selection. This technique works on a cohort level rather

than in individual tumours, and ignores selection due to copy number alterations. b I An example cohort comprising

tumours with varying evolutionary histories. c I $\mathrm{dN} / \mathrm{dS}$ identifies clonal and subclonal selection of driver events within

cancer genes. However, this approach is unable to infer selection within individual clones, and so the group of tumours

with no subclonal selection is not identified.

Previous negative selection is identified, as would be expected within genes

essential for cellular function. Passenger mutations are not under positive or negative selection. 


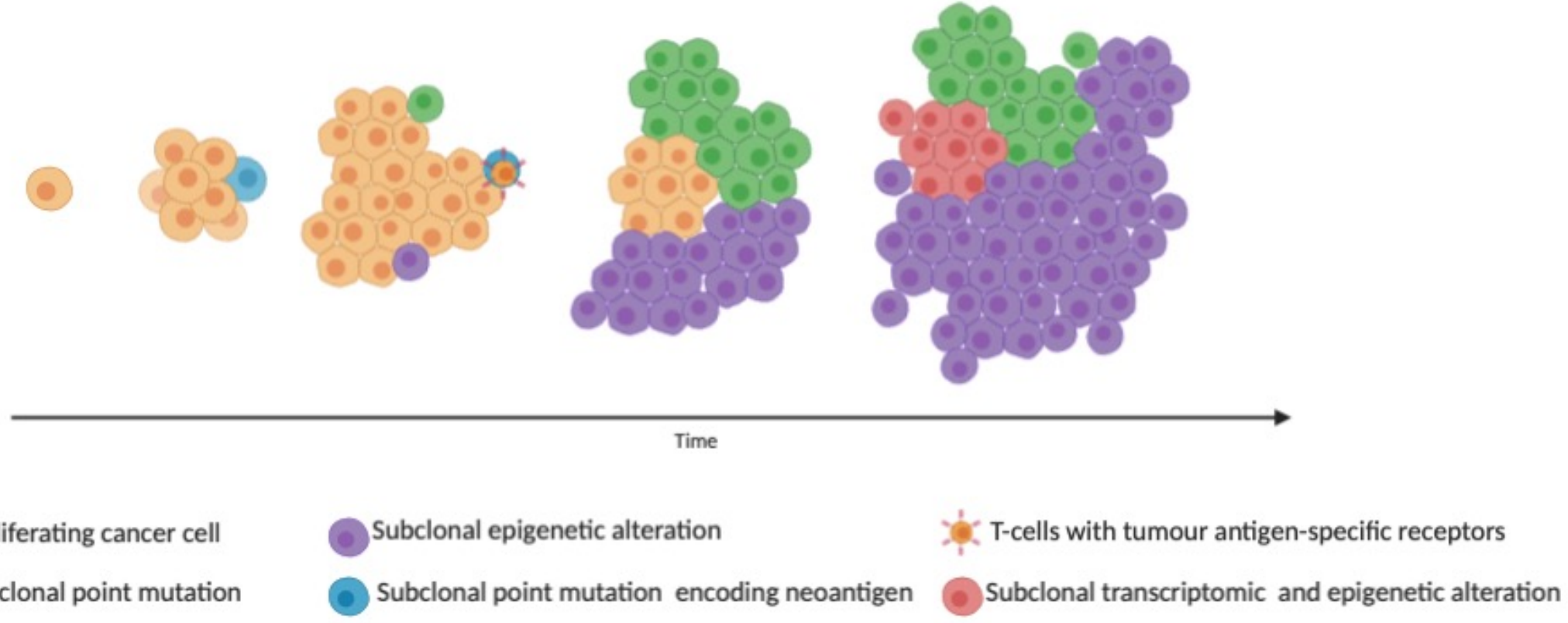

Fig. 4 | Tumour evolution may be incorrectly classified using an exclusively genomic approach. The evolving tumour acquires traits conveying selective advantages. In this example, subclones arise that contain genetic, epigenetic or transcriptomic alterations that give a selective advantage. The subclone containing an advantageous epigenetic alteration begins a selective sweep of the tumour. The subclone containing a neoantigen that stimulates the anticancer immune response is eliminated by neoantigen- reactive $T$ cells. Here, an exclusively genomic approach would fail to identify all of the functional events shaping the evolution of this tumour. 


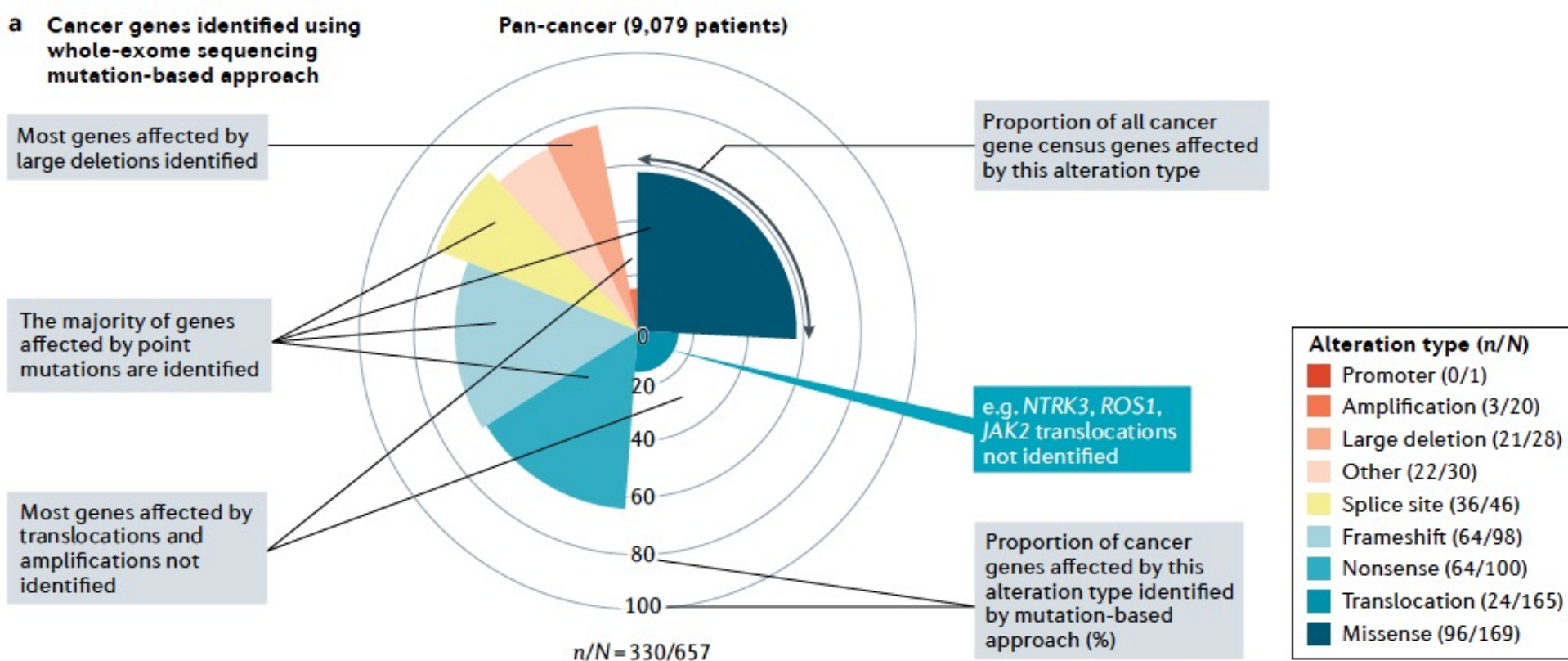

b Cancer genes identified using whole-exome sequencing mutation-based approach in selected cancer types BRCA (779 patients) renction pationts)

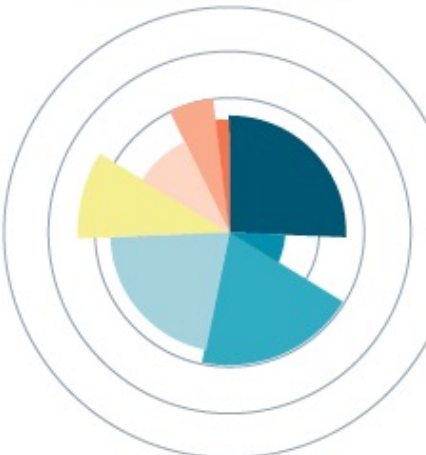

$n / N=51 / 98$

\begin{tabular}{|} 
Alteration type $(n / \mathrm{N})$ \\
Amplification $(1 / 2)$ \\
Large deletion $(3 / 5)$ \\
Other $(4 / 9)$ \\
Splice site $(6 / 9)$ \\
Frameshift $(11 / 21)$ \\
Nonsense $(11 / 19)$ \\
Translocation $(2 / 8)$ \\
Missense $(13 / 25)$ \\
\hline
\end{tabular}

LGG (510 patients)

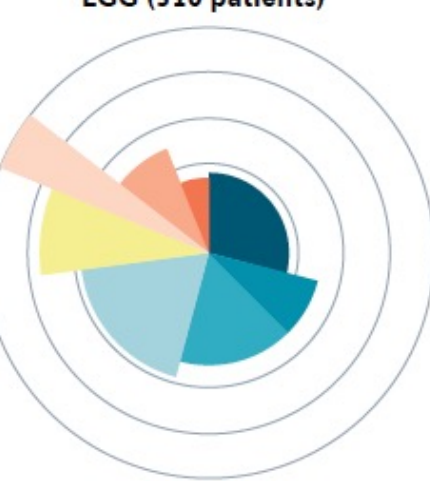

$n / N=24 / 48$

Alteration type $(n / N)$

Altoration typo (n/N)

Amplification (1/3)

Large deletion (2/4)

Other (2/2)

Splice site (3/4)

- Frameshift (5/9)

Nonsense (4/8)

Translocation (2/4)

Missense (5/14)

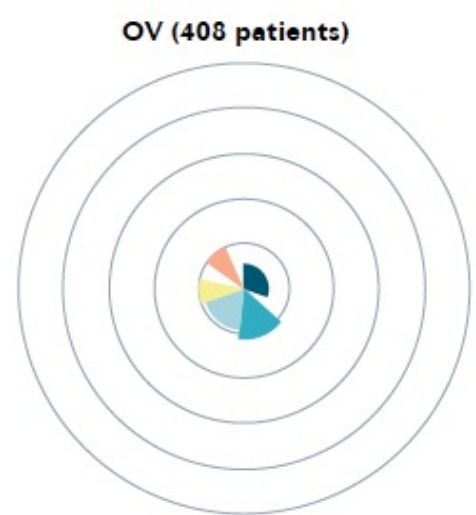

$n / N=8 / 60$

Alteration type $(n / N)$

Amplification (0/4)

Large deletion $(1 / 5)$

Large deletion $(0 / 4)$

Splice site (1/5)

Frameshift (2/11)

Nonsense (2/9)

Translocation (0/4)

Translocation (0/4)
Missense (2/18)
Alteration type $(n / N)$

Amplification (0/4)

Large deletion (1/1)

Splice site $(1 / 4)$

Frameshift $(2 / 8)$

Nonsense (2/6)

Translocation (1/41)

Missense (2/13)
Fig. 5 I Comparison of cancer genes defined by the COSMIC Cancer Gene Census and by a systematic pan-cancer whole-exome sequencing

mutation-based approach. The COSMIC Cancer Gene Census 60 curates lists

of 'Tier 1' cancer driver genes that have a cancer- related functional role as well as documented evidence of recurrent cancer- causing mutations, and the types of alteration that affect these genes. a I Chart displaying the alteration types affecting COSMIC (release v90) cancer genes that overlap with cancer genes identified in a systematic mutation- based approach by Bailey et al.59 based on pan- cancer whole- exome sequencing. In this analysis, genes that were rescued following manual curation by Bailey et al.59 were excluded, and only genes listed in COSMIC as affecting cancer types studied by Bailey et al.59 were considered. In some cases, COSMIC cancer genes are included more than once if they are affected by multiple alteration types. $\mathrm{n}$ is the number of cancer genes that were identified by the approach of Bailey et al.59, while $\mathrm{N}$ is the total number of COSMIC cancer genes. With the whole- exome sequencing mutation- based approach, many COSMIC cancer genes were not identified, in particular, those affected by translocation and amplification. This underlines the importance of the role of cancer genes that are not frequently affected by exonic mutations as functional driver events in cancer. The systemic approach of Bailey et al. identified the majority of genes affected by large deletions; in such genes, there may be functional overlap of deletions and loss- of- function mutations, and so they may be more easily identified as drivers. Clearly,

COSMIC lists are not exhaustive, and it is probable that many important driver events have not yet been identified. b I For four selected cancer types included in the analysis by Bailey et al.59, the proportion of COSMIC cancer type- specific driver genes that were identified by a cancer type- specific whole- exome

sequencing mutation- based analysis is shown. In the example, breast invasive carcinoma (BRCA) and brain lower grade glioma (LGG) have a relatively large proportion ( $52 \%$ and $50 \%$, respectively) of known cancer type- specific cancer genes identified by the systematic mutation- based approach, whereas in ovarian serous cystadenocarcinoma (OV) and sarcoma (SARC) many known cancer genes are missed (13\% and $12 \%$ identified, respectively). Cancer genes with translocations and amplifications are frequently missed; in sarcoma, for example, only 1 of 41 COSMIC cancer genes affected by a translocation is identified by Bailey et al.59. Data for each cancer type included in the analysis by Bailey et al. are shown in Supplementary Fig. 1. 


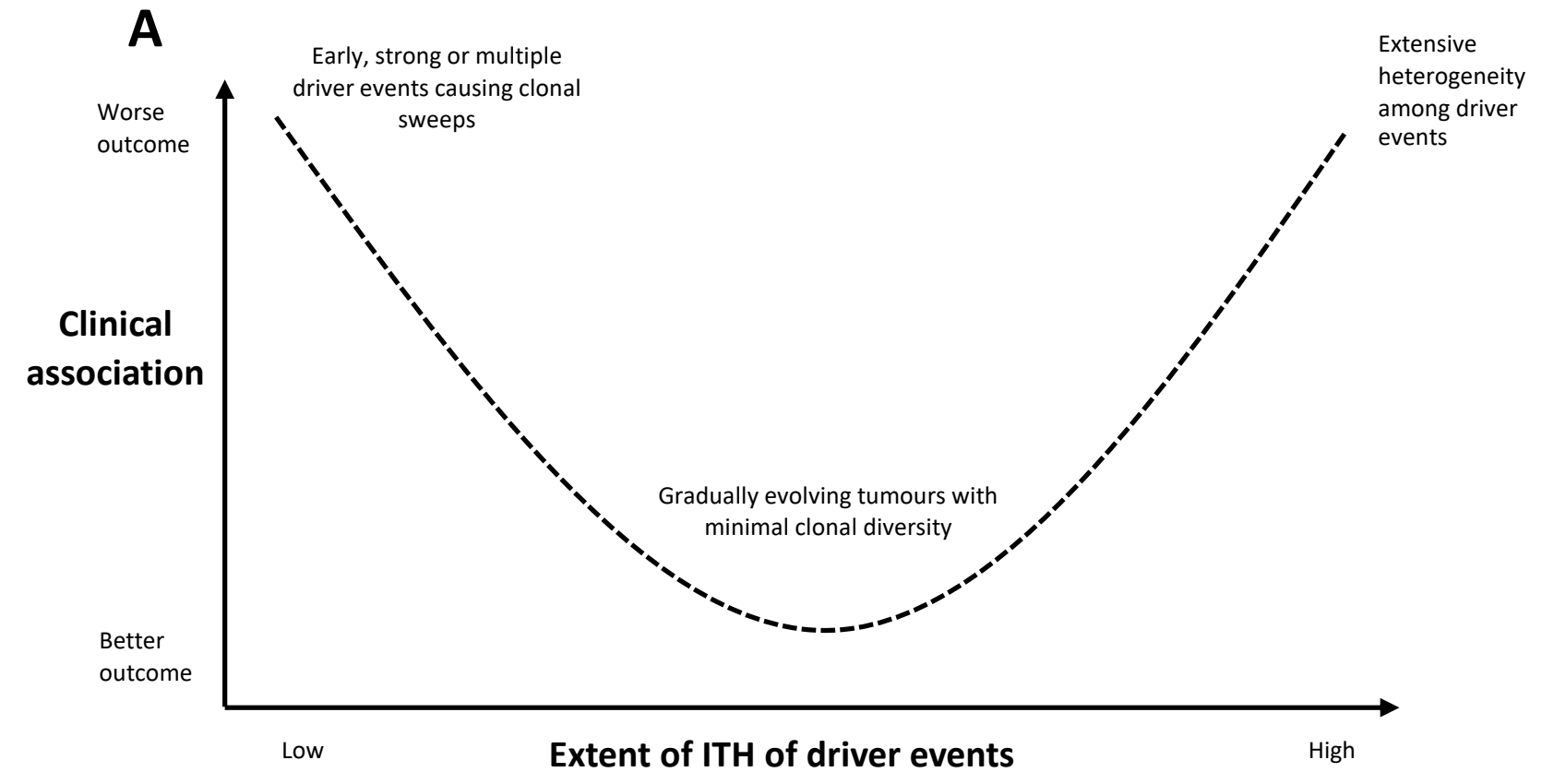

B

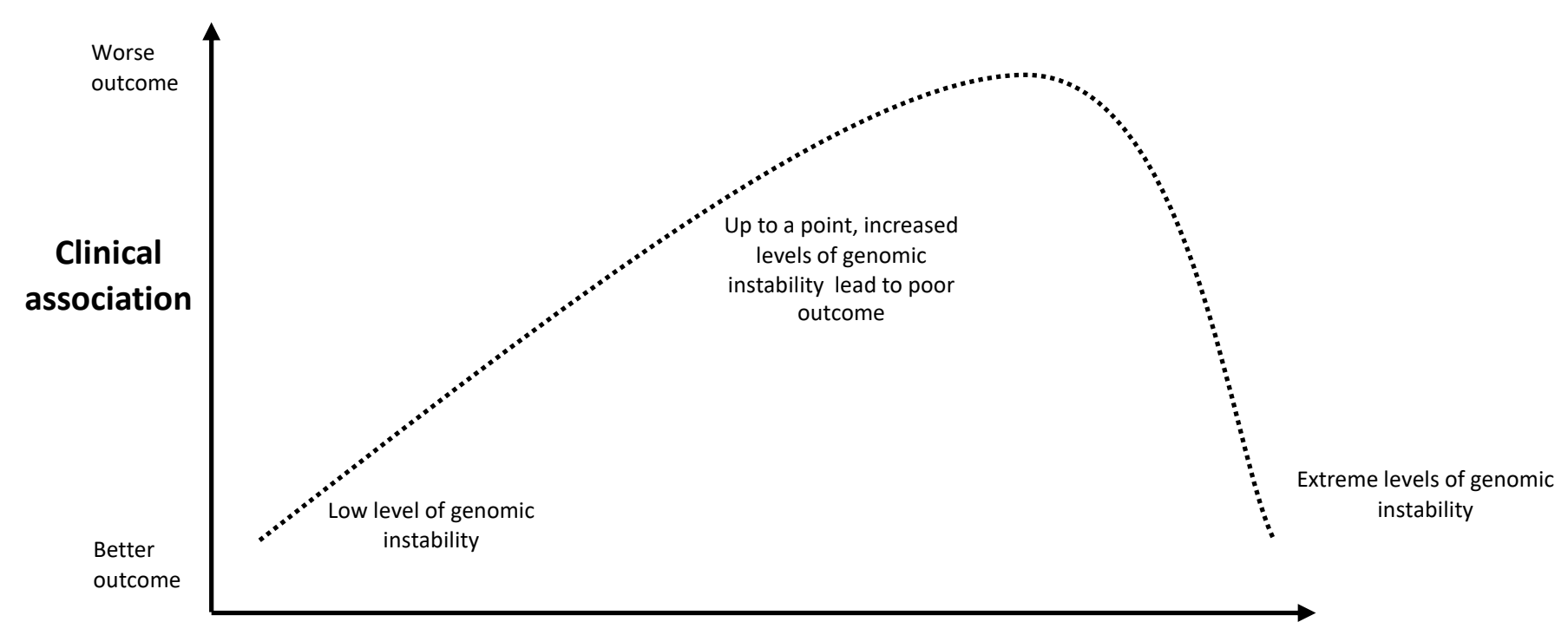

Fig. 6 | Prognostic impact of intra-tumour

heterogeneity. The relationship between intra-

tumour heterogeneity (ITH)

and clinical outcome is complex, and is influenced

by the degree of clonal diversity among driver

events within the tumour

as well as the extent of genomic instability.

Hypothesized relationships between ITH of driver events and clinical outcome

(part a) and between genomic instability and clinical outcome (part b). 
